HBTEE

PORTIONS OF THIS REPQRT ABE LLEGIBLE.

It has been reproduced from the best available copy to permit the broadest rassibie avallability.

CONF- $8406156--4$

\title{
CHAOS NEAR THE COULOMB BARRIER? -- NUCLEAR MOLECULES
}

M. R. Strayer

Oak Ridge National Laboratory

Oak Ridge, Tennessee 37831

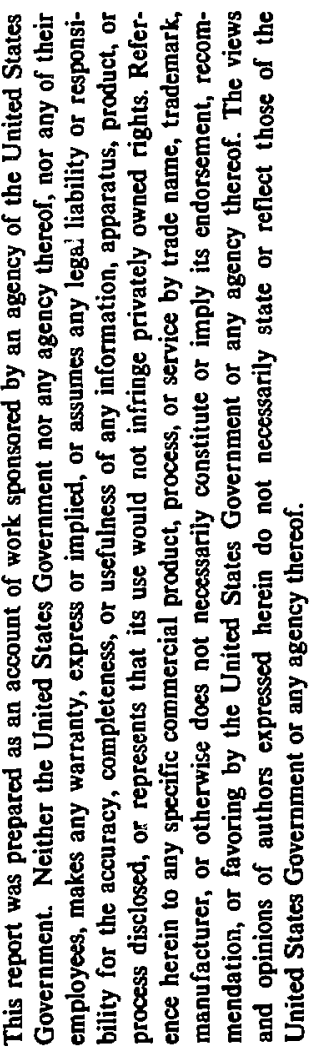

Invited Paper Presented at

International Conference on Fusion Reactions

Below the Coulomb Barrier

Combridge, Massachusetts

June 13-15, 1984

By acceptance of this article, the publisher or recipient acknowledges the U.S. Government's right to retain a nonexclusive, royalty-free license in and to any copyright covering the article.
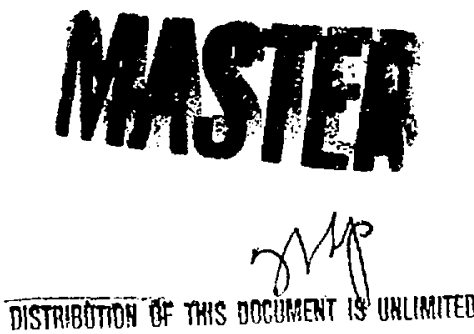


\author{
M. R. Strayer \\ Oak Ridge National Laboratory* \\ Oak Ridge, Tennessee 37831
}

\title{
I. INTRODUCTION
}

Chaos addresses in part the long-time behavior of non-integrable mechanical systems and how such systems, even though completely deterministic, develop a degree of randomness. 1 These concepts have been known for over 100 years $^{2}$ and were first addressed in the context of nuclear physics by Fermi, Pasta, and Ulam ${ }^{3} 30$ years ago. In present-day theoretical nuclear physics, the long-time mechanical behavior of nuclear systems arises through the various classical and semi-classical limits of the quantum many-body problem as, for example, in siudies of the time-dependent HartreeFock (TDHF) approximation, ${ }^{4}$ the nuclear partition function, 5 fission 1 ifetimes, ${ }^{6}$ and S-matrix elements. ${ }^{7}$ There is presently considerable interest in learning how to requantize these types of classical motion $8-12$ and obtain a viable approximation to the quantum spectrum.

Hydrodynamic models of matter in heavy-ion reactions exhibit the characteristic behavior of dissipative fluid flow, ${ }^{13}$ and details of the momentum transfer and particle multiplicities may be suggesting the formation of attractor regions in the reaction phase space. ${ }^{14}$ Isoscalar and isovector heavy-ion monopole and quadrupole giant resonances have been computed in ${ }^{16} \mathrm{O}$ and ${ }^{40} \mathrm{Ca}$ by examining the quasiperiodic motion of these systems using the TOHF approximation.15 These calculations yield unquantized vibrational frequencies in good agreement with both the RPA and the GCM methods and have the classical interpretation of being the most probable frequencies of the RPA strength functions. Using the same method, Weiss ${ }^{16}$ studied the deepinelastic fragments of the ${ }^{40} \mathrm{Ca}+{ }^{40} \mathrm{Ca}$ collision at a laboratory bombarding energy of $400 \mathrm{MeV}$. He computed large amplitude structures laying at energies of about $50 \mathrm{MeV}$ in excitation energy and suggested that these structures have many of the properties of simple giant resonances.

Other authors have addressed the question of quasiperiodic cluster and molecular structures in both light ${ }^{17-19}$ and heavy 20-22 nuclei. In light nucleus collisions the long-time quasiperiodic motion of $\alpha$-like clusters gives rise to qualitative agreement between theory and experiment ${ }^{23,24}$ and has stimulated a variety of experimental ${ }^{25}$ and theoretical ${ }^{26}$ investigations searching for similar phenomena in heavy nuclei. All of

F"...th' whole worl's in a terrible state o'chassis!", S.0'casey, Act, II Juno and the Paycock. under contract DE-AC05-840R21400 with Martin Marietta Energy Systems, Inc. 
the TOHF work seems to predict the existence of highly nonlinear structures embedded in the dynamics of heavy-ion reactions. The present work examines in detail the classical behavior of the $\alpha+{ }^{14} \mathrm{C}$ and the ${ }^{12} \mathrm{C}+{ }^{12} \mathrm{C}\left(\mathrm{O}^{+}\right)$collision at energies near the Coulomb barrier. The long-time motion of the compound nuclear system is identified in terms of its classical quasiperiodic and chaotic behavior. The consequences of this motion are discussed and interpreted in terms of the evolution of the system along a dynamical energy surface.

\section{TOHF FORMALISM}

The theoretical foundations of the nuclear TDHF formalism have been extensively reviewed in the literature, 27-29 and only a brief discussion will be presented. The most comprehensive treatment of the basic material is given in Ref. 28 . We follow the treatment of Kerman and Koonin" who obtain the TDHF equations from the Schwinger variational principle 30 for the many-body action $S_{2}(\operatorname{set} \hbar=1)$

$$
S=\int_{t_{1}}^{t_{2}} d t\left\langle\Psi(t)\left|i \partial_{t}-H\right| \Psi(t)\right\rangle
$$

in terms of the Hamiltonian of the system

$$
H=\sum_{i=1}^{A} T_{i}+\frac{1}{2} \sum_{i, j=1}^{A} V_{i j},
$$

where $K$ is the one-body kinetic energy operator and $V$ is the two-body interaction, and where $\Psi(t)$ is the wavefunction of the A-nucleon system which is assumed to have the form of a Slater determinant of time-dependent single-particle states $\Psi_{\lambda}(t)$

$$
\Psi\left(\vec{r}, \ldots . \vec{r}_{A} ; t\right)=\frac{1}{\sqrt{A !}} \operatorname{det}\left\|\psi_{\lambda_{i}}(\vec{r}, t)\right\| \text {. }
$$

Equations of motion are obtained

$$
\begin{aligned}
& i \dot{\psi}_{\lambda}(\vec{r}, t)=\frac{\delta\langle H\rangle}{\delta \psi_{\lambda}^{*}(\vec{r}, t)} \\
& i \dot{\psi}_{\lambda}^{*}(\vec{r}, t)=\frac{-\delta\langle H\rangle}{\delta \psi_{\lambda}(\vec{r}, t)}, \quad \lambda=1, \ldots, A
\end{aligned}
$$

An independent functional variation with respect to $\psi_{\lambda}$ and $\psi_{\lambda}^{\star}$, where

$$
\langle H\rangle=\langle\Psi(r)|H| \Psi(t)\rangle \text {. }
$$

The Eqs. (4) leave the action (1) stationary and the function variation

$$
h(\vec{r}, t) \psi_{\lambda}(\vec{r}, t)=\frac{\delta\langle H\rangle}{\delta \psi_{\lambda}^{*}(\vec{r}, t)}
$$

defines the Hartree-Fock one-body Hamiltonian. These equations allow an interpretation in terms of classical field coordinates $\phi_{\lambda}(\vec{r}, t)$ and momenta $\pi_{\lambda}(\vec{r}, t)$, 


$$
\begin{aligned}
& \phi_{\lambda}=\left(\psi_{\lambda}+\psi_{\lambda}^{*}\right) / \sqrt{2} \\
& \pi_{\lambda}=\left(\psi_{\lambda}-\psi_{\lambda}^{*}\right) / \sqrt{2} i
\end{aligned}
$$

which yield Hamiltonian's equation for the field $\phi_{\lambda}$ and its conjugate momentum $\pi_{\lambda}$,

$$
\begin{aligned}
& \dot{\phi}_{\lambda}(\vec{r}, t)=\delta\langle H\rangle / \delta \pi_{\lambda}(\vec{r}, t) \\
& \dot{\pi}_{\lambda}(\vec{r}, t)=-\delta\langle H\rangle / \delta \phi_{\lambda}(\vec{r}, t) .
\end{aligned}
$$

Equations (4) are solved on a 3-D space time lattice ${ }^{30}$ for a series of initial conditions specified by

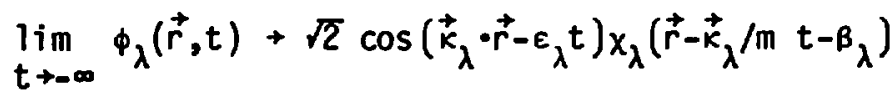

and

$$
\lim _{t \rightarrow-\infty} \pi_{\lambda}(\vec{r}, t)+\sqrt{2} \sin \left(\vec{k}_{\lambda} \cdot \vec{r}-\varepsilon_{\lambda} t\right) x_{\lambda}\left(\vec{r}-\vec{k}_{\lambda} / m t-\beta_{\lambda}\right)
$$

where

$$
h x_{\lambda}(\vec{r})=\varepsilon_{\lambda} x_{\lambda}(\vec{r}), \quad \lambda=1, \ldots, A .
$$

are solutions to the initial static Hartree-Fock equations. We solve both the static and the dynamic Hartree-Fock equations, assuming axial symmetry in cylindrical polar coordinates. Details of the method are given in Ref. 30, and we employ the local finite-range 31 (BKN) form of the Skyrme force without any spin-orbit forces. The choice of the BKN force is motivated in part by our desire for a realistic treatment for vibrational motion in light nuclei and the known failure ${ }^{32}$ of the nonlocal Skyrme forces to describe harmonic vibrations in these nuclei.

The wavepacket initialization, Eq. (9), leads to a large class of dynamic behavior, depending on the parameters $\kappa_{\lambda}$ and $\beta_{\lambda}, \lambda=1, \ldots . A$, labeling the initial state. In the present work we consider binary fragments fixed in the center of mass, separated along the symmetry axis by a given displacement $R_{0}$ and having a kinetic energy of relative motion, $E_{0}$. Thus, even though we are not primarily concerned with collisional phenomena, the initial state has a configuration which is similar to a semiclassical entrance channel.

We consider the long-time classical behavior of the A particle system which evolves from the initial state, Eq.'(9), using the TDHF, Eqs. (8). We characterized the properties of the system in terms of the isoscalar $(I=0)$ and isovector $(I=1)$ density,

$$
\hat{\rho}_{I}(\vec{r})=\sum_{\lambda=1}^{A} \delta\left(\vec{r}-\vec{r}_{\lambda}\right) \hat{a}_{I}(\lambda)
$$

with 


$$
\left\langle q\left|\hat{a}_{I}\right| q^{\prime}\right\rangle= \begin{cases}\delta_{q q^{-+1}}, & q=\text { proton } \\ (-1)^{I} \delta_{q q^{\prime}}, & q=\text { neutron. }\end{cases}
$$

which is consistent with the previously stated classical interpretation. The density

$$
\rho_{I}(\vec{r}, t)=\left\langle\psi(t)\left|\hat{\rho}_{I}(\vec{r})\right| \Psi(t)\right\rangle,
$$

in terms of the real fields $\pi_{\lambda}$ and $\phi_{\lambda}$ is

$$
\rho_{I}(r, t)= \begin{cases}\rho_{p}(\vec{r}, t)+\rho_{n}(\vec{r}, t) & I=0 \\ \rho_{p}(\vec{r}, t)-\rho_{n}(\vec{r}, t) & I=1\end{cases}
$$

with

$$
\rho_{q}(\vec{r}, t)=\frac{1}{2} \sum_{\lambda}\left[\pi \lambda_{\lambda, q}(\vec{r}, t)^{2}+\phi_{\lambda}(\vec{r}, t)^{2}\right], q=p, n
$$

The gradient iteration method of solution of the lattice equation ${ }^{33}$ is equivalent to the first-order imaginary time propagator ${ }^{34}$ with the inclusion of a kinetic energy dampening operator and gives finite difference equations at lattice points $r_{j}$ and $z_{j}$

$$
\begin{array}{ll}
r_{i}=\Delta r\left(i-\frac{1}{2}\right) & i=1,2, \ldots N_{r} \\
z_{j}=\Delta z(j-1) & j=1,2, \ldots N_{z}
\end{array}
$$

as

$$
\psi_{\lambda}^{(N+1)}(i j)=0\left\{\psi_{\lambda}^{(N)}(i j)-\varepsilon D\left(k_{0}\right)\left(W_{\lambda}^{(N)}-\varepsilon_{\lambda}^{(N)}\right) \psi_{\lambda}^{(N)}(i j)\right\}
$$

where $w_{\lambda}^{(N)}, \varepsilon_{\lambda}^{(N)}$, and $\psi_{\lambda}^{(N)}(i j)$ are respectively the Hartree-Fock one-body Hamiltonian, the single-particle energy and wavefunction at the Nth iteration. The operation of Schmidt orthogonalization of the wavefunction at each structure is denoted by the operator 0 . In Eq. (13) $D\left(k_{0}\right)$ is the kinetic energy damping operator

$$
D\left(k_{0}\right)=\left\{1+T / k_{0}\right\}^{-1} \text {, }
$$

where $T$ is the kinetic energy operator defined at the lattice point. Details of the method are given elsewhere. 35 In $\mathrm{Eq} .(13) \varepsilon \simeq 0.05$ is the imaginary time parameter and $k_{0} \approx 40 \mathrm{MeV}$ fixes the energy damping scale. The convergence of the gradient iteration method is shown in Fig. 1 for three different nuclear configurations, ground state ${ }^{20 \mathrm{Ne}}$, ground state ${ }^{12} \mathrm{C}$, and the shape isomeric HF solution for carbon ${ }^{12} \mathrm{C}\left(\mathrm{O}^{+}\right)$. The fractional change in the Hartree-Fock energy, $\Delta E^{(N)}$,

$$
\Delta E^{(N)}-\frac{E^{(N+1)}-E^{(N)}}{E^{(N)}}
$$




$$
\tau_{N}=\mathbf{i} \in \mathbf{N} \text {. }
$$

All of the solutions shown converge to within 1 in $10^{9}$ after 100 iterations. This rate of convergence is not as great as that reported in Ref. 33, however we have employed a slightly different treatment of the dampening operator and have treated the imaginary time step parameter, $\Delta \tau$, as a constant, independent of the iteration sequence. The HF iteration sequence is fully self-consistent for all of the systems shown in Fig. 1. The structure in the curve for ${ }^{20} \mathrm{Ne}$ results from a reordering of the single-particle states due to three separate shell crossings. The exponential convergence of the energy is characteristic of the damped gradient iteration method throughout the periodic table.

The density constraint method is easily incorporated into the formation of the Hartree-Fock equations using the Schwinger method ${ }^{4}$ by the replacement in Eq. (8),

$$
H \rightarrow H+\int d^{3} r F(\vec{r}) \rho(\vec{r}),
$$

where $\rho(\vec{r})$ is the HF one-body density, $\rho_{c}(\vec{r})$ is the density function we wish to obtain as our solution of the HF problem, and $F(r)$ is an auxiliary field which must drive the system so that

$$
\rho(\vec{r})+\rho_{c}(\vec{r})
$$

during the iteration process. The field $F(\vec{r})$ is a regular $L_{2}$ function, and thus the addition of the field term in Eq. (17) does not alter the characteristics of the spectrum of $\mathrm{H}^{34}$ The modification of the gradient iteration method to include the density as a constraint is straightforward, assuming that the HF single-particle states at iteration $N, \psi^{(N)}$ gives the density $\rho_{C}$ approximately

$$
\rho^{(N)}(\vec{r})=\sum_{\lambda}\left|\psi_{\lambda}^{(N)}(\vec{r})\right|^{2} \sim \rho_{c}(\vec{r})
$$

then the field at iteration $F^{(N+1)}$ which forces $\rho^{(N+1)}$ to be $\rho_{c}$ can be evaluated in perturbation theory and results in the set of modified gradient iteration equations,

$$
\begin{gathered}
\bar{\psi}_{\lambda}(\vec{r})=0\left\{\psi_{\lambda}^{(N)}(\vec{r})-\varepsilon D\left(k_{0}\right)\left[w_{\lambda}^{(N)}+F^{(N)}-\varepsilon_{\lambda}^{(N)}\right] \psi_{\lambda}^{(N)}(\vec{r})\right\} \\
F^{N+1)}(\vec{r})=F^{(N)}(\vec{r})+c_{0} \frac{\delta \rho(\vec{r})}{0_{0}+2 \varepsilon \rho(N)}(\vec{r})
\end{gathered}
$$

with

$$
\delta \rho(\vec{r})=\sum_{\lambda}\left|\bar{\Psi}_{\lambda}(\vec{r})\right|^{2}-\rho^{(N)}(\vec{r})
$$

and

$$
\psi_{\lambda}^{(N+1)}(\vec{r})=0\left\{\bar{\psi}_{\lambda}(\vec{r})-\varepsilon\left[F^{(N+1)}(\vec{r})+\delta f(\vec{r})\right] \bar{\psi}_{\lambda}(\vec{r})\right\}
$$




$$
\delta f(r)=C_{0} \frac{\rho^{(N)}(r)-\rho_{c}(\vec{r})}{D_{0}+2 \varepsilon \rho_{c}(\vec{r})} .
$$

The parameters $C_{0}$ and $D_{0}$ must be included to insure stability of the numerical method. In practice, we choose $C_{0} \approx 0.9$ and $D_{0}$ as $7 \times 10^{-5}$. The details of the convergence of the method does not appear to be sensitive to this choice. A complete discussion of the method is given in Refs. 33 and 35.

\section{QUASIPERIODIC SOLUTIONS}

We have studied quasiperiodic motion in two nuclear systems, 180 and $24 \mathrm{Mg}$, which experimentally display a type of molecular structure. We consider 180 as an 18particle determinant of single-particle states which are "close" both in terms of energetics and in terms of collective and shape variables to an $\alpha+{ }^{14} \mathrm{C}$ configuration. Experimentally, the $\alpha$-induced $\gamma$ decays of ${ }^{18} 0$ have been measured in the ${ }^{14} C(\alpha, \gamma)^{18} 0$ radiative capture reactions. ${ }^{24}$ This work reports enhanced electric dipole and quadrupole transitions and suggests the existence of a "molecular band" built on the 3.63 $0^{+}$state. This state has been extensively studied theoretically ${ }^{36}$ and has the structure of a $(4 p-2 h)$ core polarized deformed state. The formation of an a cluster molecular configuration in ${ }^{18} 0$ has been suggested in terms of a group theoretical algebraic approach, 37 in with the structure of the molecular states are constructed from $S$ and $P$ bosons and have a $U(4)$ symmetry.

The present calculations address this question from a microscopic "view" which provides both a geometrical (classical) picture of the molecule, as well as characteristic information on the rotational and vibrational structure of the molecule. The TOHF Eqs. (4) are solved to determine the time evolution of the ${ }^{18} 0$ system from an initial state comprised of a ground state ${ }^{4} \mathrm{He}$ nucleus and a ground state ${ }^{14} \mathrm{C}$ nucleus having an initial separation of $15 \mathrm{fm}$ and a kinetic energy of relative motion of $7.5 \mathrm{MeV}$ in the center of mass. ${ }^{19}$ Thes 2 are axially symmetric head-on collisions which initially translate along classical Coulomb trajectories. The density contours for the subsequent motion are shown in Fig. 2. The initial contact occurs at about $40 \mathrm{fm} / \mathrm{C}$, and for the times shown in $\mathrm{Fig} .2$, the system has relaxed into a configuration undergoing highly nonlinear quasiperiodic notion. The density distribution has the appearance of an $\alpha$-particle cluster and a carbon cluster which are exchanging with one another about the $\mathrm{CM}$ of the ${ }^{18} 0$ system. This type of clustering in TDHF calculations arises in part from the strong S-wave components of the density-dependent force, which favors spin-isospin symetric configurations, and in part from the unusual stability of the ${ }^{4} \mathrm{He}+{ }^{14} \mathrm{C}$ initial configuration.

The initial ${ }^{14} \mathrm{C}$ nucleus is treated in the filling approximation in which four protons uniformly fill the entire p-shell, thus giving an approximation to ${ }^{14} \mathrm{C}$ which yields a spherically symetric state. The multipole and isospin decomposition of the density is defined with the convention 


$$
M_{\ell I}(t)=\int d^{3} r Y_{\ell 0}(\hat{r}) \rho_{I}(\vec{r}, t) r^{\ell}
$$

and

$$
M_{\ell I}(\omega)=\int_{-\infty}^{\infty} d t e^{-i \omega t} M_{\ell I}(t)
$$

The time and frequency dependence of the isoscalar, quadrupole and octupole moments, and the isovector dipole moment are shown in Fig. 3, where we observe well-developed oscillations with clear frequencies for times as long as $3 \times 10^{3} \mathrm{fm} / \mathrm{c}$ (about $10^{-20}$ $\mathrm{sec})$. These are times which are long compared to heavy-ion reaction times for this sy'stem. The isoscalar octupole mode corresponds to a relatively plire 4-MeV frequency and the isoscalar quadrupole to a mixture of 8 and $15 \mathrm{MeV}$ frequencies. The isovector mode is a combination of 4-MeV and 9-MeV frequencies. The observation of the 4-MeV frequency in both the isoscalar octupole and isovector dipole channels is probably due to a single mode occurring in both projections.

The most extensive and compeliing experimental evidence for the existence of nuclear molecules lies in the narrow resonances observed in the ${ }^{12} \mathrm{C}+{ }^{12} \mathrm{C}$ reactions near the Coulomb barrier. ${ }^{17}$ A large number of experimental data has been amassed, and the most successful organization of these data is in terms of the U(4) model of Iachello. ${ }^{23}$ The representation of the data in terms of this model suggests that the molecule involves a structure related to the $7.08 \mathrm{MeV} 0^{+}$state of ${ }^{12} \mathrm{C}$. This state has a well-established cluster structure and is approximately given as a HF shape isomer of ${ }^{12} \mathrm{C} .18$ The energy as a function of deformation and the density contours for both the ground state ${ }^{12} \mathrm{C}$ and the carbon isomer are shown in Fig. 4. The density contours corresponding to the time evolution from an initial configuration based on these two states are shown in Fig. 5(a) and 5(b). The initial state is also labeled by $C M$ energy, $E_{c m} \approx 7.5 \mathrm{MeV}$ and separation along the axis of symmetry, $R_{s} \approx 12 \mathrm{fm}$. Again, these are head-on collisions in which the matter flow undergoes a complex pattern of quasiperiodic motion. The contours in Fig. $5(a)$ are those for the isoscalar density, while those in Fig. 5(b) are for the motion of the isovector density. For the forces used in this study, the structure in the isovector density arises solely through the Coulomb force. While the motion of the isoscalar density appears regular, the isovector density has a complex high multipolar structure which varies rapidly on a time scale where the isoscalar density varies slowly.

The corresponding long-time behavior of the system is shown in Fig. 6 for the isovector dipole and the isoscalar quadrupole, octupole, and RMS radius (two times the physical radius of ihe system).

The time and frequency dependence of the moments is qualitatively similar to that of the 180 system but quantitatively very different. The time scales are much longer in Fig. 6; the ${ }^{12} \mathrm{C}\left(\mathrm{O}^{+}\right)+{ }^{12} \mathrm{C}$ system has been followed in for almost $5 \times 10^{-20}$ $s e c$, about a fartor of 5 longer than the ${ }^{18} 0$ system. The time dependence of the isoscalar octupole motion is very regular and corresponds to a low-frequency 
vibration of about $1.5 \mathrm{MeV}$. As before, the isoscalar quadrupole motion is a combination of low- $(\sim 2.5 \mathrm{MeV})$ and high- $(\sim 8 \mathrm{MeV})$ frequency motion.

The individual motion of the proton and neutron fluids give a slightly different perspective on the motion shown in Fig. 7 and the frequency behavior of the moments of the neutron and proton densities for ${ }^{24} \mathrm{Mg}$. Here, because of the long scale, we see a very complex pattern of small-amplitude oscillations in the motion of the system. This snall-amplitude frequency behavior is indicative of the breakdown of periodicity in the system.

The motion of both the ${ }^{18} 0$ and the ${ }^{24} \mathrm{Mg}$ can also be described in terms of collective variables and in terms of collective energies. As the system evolves along a TDHF path, the density, which may be thought of as a collective variable of the system, undergoes a complex motion. The corresponding potential energy as a function of the density can be calculated using the density-constrained Hartree-Fock (DCHF) equations. Using this method, we freeze the density along the TDHF path and minimize the energy of the system along the path using Eqs. (18). This energy is a function of the constraining density and is shown in Fig. 8 for the ${ }^{18} 0$, and in Fig. 9 for the $24 \mathrm{Mg}$ system, as a function of the isoscalar octupole and quadrupole degrees of freedom. The energy is a surface in the $q_{2}, q_{3}$ plane. During the time evolution, each system travels on a path in this plane. Thus, not all values of the energies in this region were observed. These plots were obtained using a least-squares fit to the HF energies along the path. The time history for the motion of the 180 system with respect to the surface in Fig. 8 is as follows. The initial solution corresponds to the coordinates $q_{2}=1.7 q_{20}, q_{3}=-2.5 q_{30^{\circ}}$. The system quickly evolves into the region shown in Fig. 8, principally following the equipotential contours, but with a drift towards small $q_{2}$. The minimum energy in Fig. 8, $E_{0}$, is an axially symmetric shape isomer of the ${ }^{18} 0$ system and is a stable solution of the uncorstrained static HF equations. A similar time history of the ${ }^{24} \mathrm{Mg}$ system can be understood in terms of Fig. 9. The initial trajectory begins at $q_{2}=1.5 q_{20}$ and $q_{3} \approx+2.9 q_{30}$, with $q_{20}=582.7 \mathrm{fm}^{2}$ and $q_{30}=3431.0 \mathrm{fm}^{3}$. The system again evolves into a region shown in Fig. 9 and traverses a complex path about the shape isomer, $E_{0}=-143.8$ at $q_{2} \approx$ $355 \mathrm{fm}$ in $q_{3} \approx 0.0$. The energy contours in Fig. 9 are the constrained energies $\Delta E=$ 2 (E(constrained) $-E_{0}$ ) in units of $\mathrm{MeV}$. The axially symetric ground state of ${ }^{24} \mathrm{Mg}$ occurs at $q_{2}=67.9 \mathrm{fm}^{2}$, with an energy of $-179.7 \mathrm{MeV}$. The general features of the energy surface as shown in Fig. 9 are in agreement with those obtained using Nilsson-Strutinsky methods. 38

\section{CHAOS}

The approach of classical systems to chaos addresses a broad spectrum of physical problems in the mechanics of fluids, ${ }^{39}$ in storage ririgs and colliding beam accelerators, 40 in plasmas, 41 and in molecular and chemical physics.42 Sidestepping the detailed questions as to the universality of bifurcating systems 43 and the topology 
of attractor phase space, we follow the general methods outlined by Koonin and Williams 12 in their treatment of requantization of semiclassical nuclear Hamiltonians. These authors study a variety of requantization schemes for the classical trajectories (TDHF) of a three-level SU(3) model.44 For this model, exact quantum-mechanical, as well as closed-form TDHF solutions, can be obtained. The resulting classical Hamiltonian is a function of two independent coordinates $\left(q_{1}, q_{2}\right)$ and momentum $\left(p_{1}, p_{2}\right)$ and is given in Fig. 10. Here $N_{E}$ is the total energy of their system and $x$ is the interaction strength as a (dimensionless). The potential energy surfaces $\left(p_{1}=p_{2}=0\right)$ as a function of $q_{1}, q_{2}$, are also shown for three values of $x$. The three values of $x$ correspond to one, two, and four minima. Hamiltonian equations were integrated numerically to give the trajectories and Poincaré sections in phase space. Three types of classifications of motion are given: a) periodic, b), c), and d) quasiperiodic, and e) and f) stochastic. The conclusions they put forth are quite interesting; they conclude it is not possible to requantize the classical motion whenever it is stochastic, and that a variety of methods all give reasonable solutions in the quasiperiodic regime.

The Poincaré projects for the motion in ${ }^{24} \mathrm{Mg}$ analogous to those shown in Fig. 10 are displayed in Fig. 11 for the isoscalar quadrupole and octupole modes and for the isovector dipole mode. We see that the motion is at best quasiperiodic and appears to be filling almost all of the phase space in this regime. The corresponding autocorrelation functions, defined in Eq. (20) as

$$
c_{\ell I}(\tau)=\int_{-\infty}^{\infty} \frac{d \omega}{2 \pi} e^{+i \omega \tau} M_{\ell I}(\omega)
$$

are shown in Fig. 12. The correlation function is small at all of the relevant frequencies, suggesting that the motion is indeed chaoiic.

In an attempt to find more periodic states for $24 \mathrm{Mg}$, we have also atsempted a different type of initialization.

Referring to Fig. 9, we follow the evolution from the same initial configuration until it reaches a point on the energy surface indicated by the " $x$ ". This part of the evolution takes approximately $700 \mathrm{fm} / \mathrm{c}$. At this point, we again minimize the energy using the DCHF procedure. This has the effect of localizing the system in the region of the isomer and "cooling" the system keeping the density fixed. This state is then used to continue the time evolution using the TDHF equations. The subsequent time and frequency dependence of the isoscalar moments are shown in Fig. 13, and for the isovector moments in Fig. 14. The time dependence of the isoscalar moments in Fig. 13 are indeed more periodic than previous?y; furthermore, the dominant frequency of the motion at low frequency now appears in all of the moments, as it must for an exact periodicity. This motion also appears in the odd- $\ell$ isovector moments shown in Fig. 14. However, the even-l multipoles appear chaotic and do not have the correct low-frequency behavior for exact harmonic motion. The autocorrelation functions, Eq. (20), are compared in Fig. 15 for the motion shown in Figs. 13 and 14 . Here we 
observe strong correlations which do not appreciably dampen in time.

\section{DISCUSSION}

We have solved for the time evolution of highly excited 180 and $24 \mathrm{Mg}$ from reaction-like entrance channels. In both cases, the details of the evolution are sensitive to parameters of the initialization, the relative kinetic energy, the fragment separation. For the ${ }^{18} 0$ system, we evolve from an $\alpha+{ }^{14} \mathrm{C}$ entrance channel, for the ${ }^{24} \mathrm{Mg}$ system we evolve from an asymptotic configuration of $12 i\left(0^{+}\right)+{ }^{12} \mathrm{C}$. The ${ }^{12} \mathrm{C}\left(0^{+}\right)$configuration is strongly related to a 3-a cluster configuration of carbon. The memory of the initial $\alpha$-clustering persists throughout the time evolution for both systems. In each case, the system rapidly evolves into the region of a shape isomer, undergoing quasiperiodic motion. The Poincaré phase space analysis and the behavior of the autocorrelation functions suggest that the motion is chaotic instead of quasiperiodic. The importance of identifying quasiperiodic instead of chaotic follows from the work of Ref. 49 where the quasiperiodic motion represents a known classical limit of the quantum spectrum whereas the chaotic motion does not. For $24 \mathrm{Mg}$, methods were employed to "cool" the motion and this resulted in quasiperiodic motion which was neither chaotic nor exactly harmonic.

In general terms, we are suggesting the existence of highly collective structures of a giant resonance nature having compound nuclear properties at excitation energies of $13 \mathrm{MeV}$ in 180 and $36 \mathrm{MeV}$ in ${ }^{24} \mathrm{Mg}$, and very large deformations for ${ }^{24} \mathrm{Mg}$. These ideas have been suggested previously by Cosman 45 and co-workers. From the point of view presented in this paper, these structures should exist throughout the periodic table and for a variety of different entrance channel configurations.

\section{REFERENCES}

1. R. H. G. Helleman, in "Chaos and Universality", Nordisk Institut für Theoretisk Atomfysik, November, 1981, p. 165.

2. H. Poincaré, Les Methodes Nouvelles de La Méchanique Céleste (Gauthier-Viiliars, Paris, 1892) [NASA translation, Washington, 1951].

3. E. Fermi,, J. Pasta, and S. Ulam, "Studies of Nonlinear Problems", Sci. Rep. LA-1940 (LASL, 1957).

4. A. K. Kerman and S. E. Koonin, Ann. Phys. (N.Y.) 100, 332 (1976).

5. A. K. Kerman and S. Levit, Phys. Rev. C 24, 1029 (1980).

6. S. Levit, J. W. Negele, and Z. Paltiel, Phys. Rev. C 22, 1979 (1980).

7. Y. Alhassid and S. E. Koonin, Phys. Rev.: C 23, 1590 (1981).

8. K.-K. Kan, J. J. Griffin, P. C. Lichtner, and M. Dworzecka, Nucl. Phys. A332, $109(1979)$.

9. R. Shankar, Phys. Rev. Lett. 45, 1088 (1980).

10. A. E. L. Dieperink and 0. Scholten, Nucl. Phys. A346, 125 (1980). 
11. D. H. Feng, R. Gilmore, and S. R. Deans, Phys. Rev. C 23, 1254 (1981).

12. R. D. Williams and S. E. Koonin, Nucl. Phys, A391, 72. (1982).

13. G. Bertsch, Nucl. Phys. A249, 253 (1975).

14. D. Ruelle, Math. Intellegencer 2, 126 (1980).

15. J. Błocki and H. Flocard, Phys. Lett. 85B, 163 (1979).

16. M. Weiss, "Time-Dependent Hartree-Fock and Beyond", ed. K. Goeke and P.-G. Reinhard (Springer-Verlag, New York 1982, p. 40.

17. D. A. Bromley, in "Resonances in Heavy-Ion Reactions", ed. K. A. Eberhärd (Springer, New York, 1981) p. 1.

18. R. Y. Cusson et al., in "Time-Dependent Hartree-Fock and Beyond", eds. K. Goeke and P.-G. Reinhard (Springer, New York, 1982) p. 22.

19. M. R. Strayer et al., Phys. Letts. 135B, 261 (1984).

20. H. Stöcker et al., Phys. Letts. 101B, 379 (1981).

21. K. T. R. Davies, K. R. S. Devi, and M. R. Strayer, Phys. Fev. Lett. 44, 23 (1980).

22. M. R. Strayer, R. Y. Cusson, H. Stöcker, J. A. Maruhn, and H. Greiner, Phys. Rey. C 28, 228 (1983).

23. K. A. Erb and D. A. Bromley, Phys. Rev. C 23, 2781 (1981).

24. M. Gai et al., Phys. Letts. 50, 239 (1983).

25. M. Gai, private communication.

26. F. Iachel10 and A. D. Jackson, Phys, Letts. 108B, 151 (1982).

27. S. E. Koonin, Progress in Particle and Nuclear Physics 4, 283 (1979).

28. J. W. Negele, Revs. Mod. Phys. 54, 913 (1982).

29. K. T. R. Davies, K. R. S. Devi, S. E. Koonin, and M. R. Strayer, in Heavy-Ion Science, ed. D. A. Bromley (Plenum, New York, 1983).

30. K. T. R. Davies and S. E. Koonin, Phys. Rev. C 23, 2042 (1981).

31. P. Bonche, S. Koonin, and J. W. Negele, Phys. Rev, C 13, 1226 (1976).

32. K.-F. Liu and G. E. Brown, Nucl. Phys. A265, 385 (1976).

33. R. Y. Cusson and P.-G. Reinhard, unpublished.

34. K. T. R. Davies et alo, Phys. Rev. C 18, 2631 (1978).

35. M. R. Strayer et al., to be published.

36. J. B. McGrory and B. H. Wildenthal, Phys. Rev. C I, 974 (1973).

37. F. Iachel10, Phys. Rev. C 23, 2778 (1981).

38. G. Leander and S. E. Larsson, Nuc7. Phys. A239, 93 (1975). 
39. G. Ahiers and R. P. Behringer, Progr. Theor. Phys. Supp1. 64, 186 (1980).

40. R. H. G. Helleman, Am. Inst. Phys. Conf. Proc., 57, 236 (1979).

41. "Intrinsic Stochasticity in Plasmas", eds. G. Laval and D. Gresillen (Les Editions de Physique, Orsay, France, 1980).

42. D. W. Noid, M. L. Koszykowski, aind R. A. Marcus, Ann. Rev. of Phys. Chem. 32 , 267 (1981).

43. M. J. Feigenbaum, J. Stat. Phys. 77, 65 (1980).

44. S. Y. Li, A. Klein, and R. M. Dreizler, J. Math. Phys. 11, 975 (1970).

45. E. R. Cosman, R. J. Ledoux, M. J. Bechera, C. E. Ordonez, and H. A. Al-Juwair, in "Resonances in Heavy-Ion Reactions", ed. K. A. Eberhard (Springer, New York, 1981) p. 112 . 


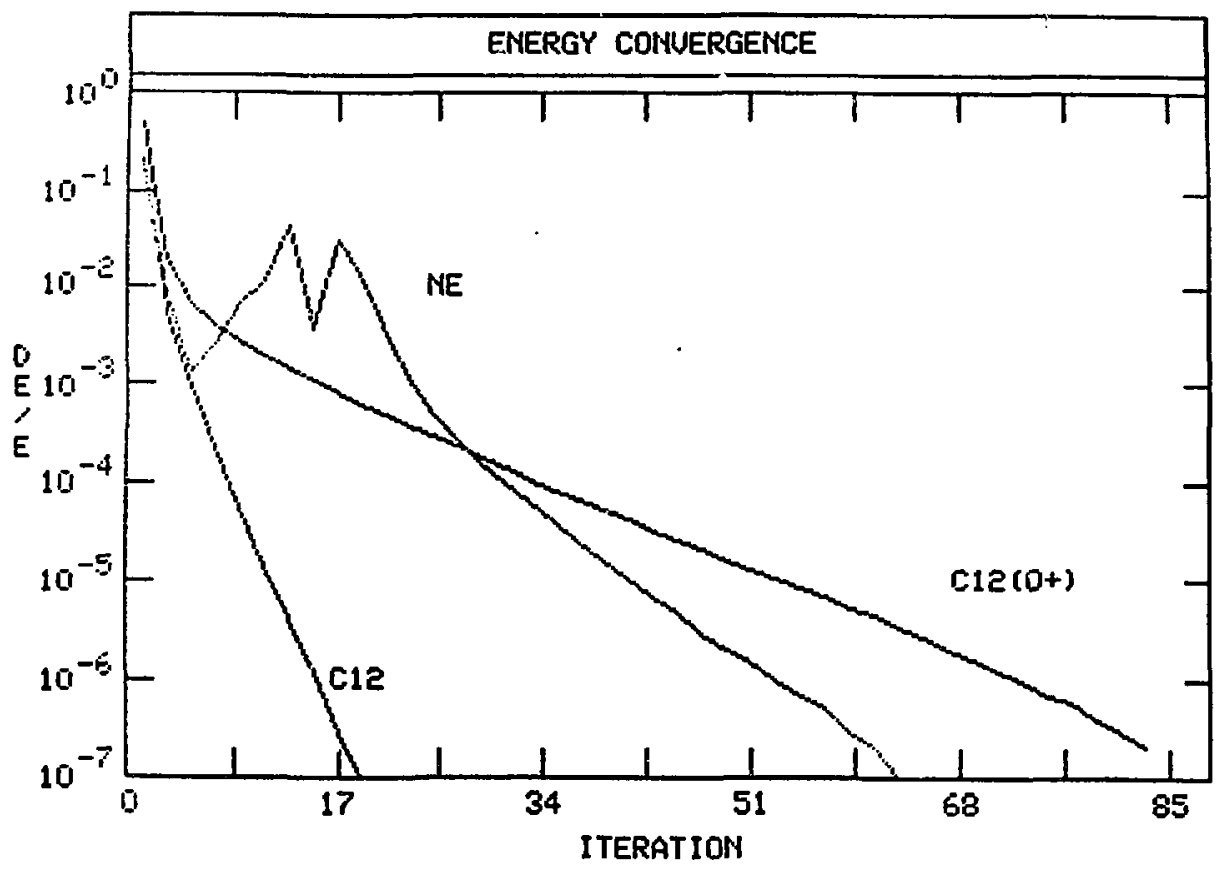

Fig. 1. Cuinvergence of the gradient iteration method for the nuclei ${ }^{12} \mathrm{C},{ }^{12} \mathrm{C}\left(\mathrm{O}^{+}\right)$ and $20 \mathrm{Ne} \Delta E^{(N)}=\left(E^{(N+1)}-E^{(N)}\right) / E^{N}$ where $E^{N}$ is the Hartree-Fock energy at the $N^{\text {th }}$ imaginary time step.

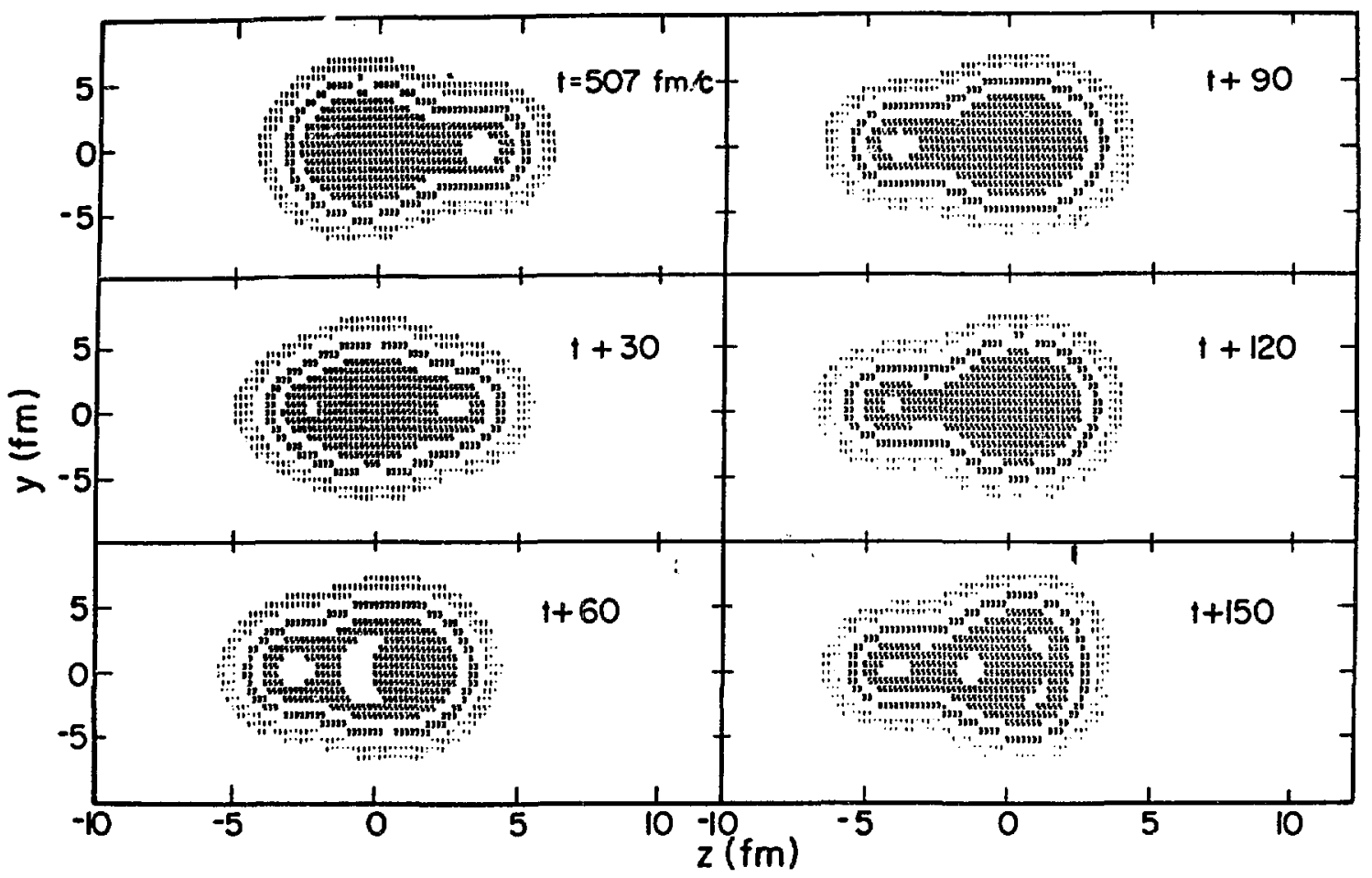

Fig. 2. Density contours $(I=0)$ in the collision plane for the ${ }^{18} 0$ system, starting from an initial $\alpha+{ }^{14} \mathrm{C}$ configuration. The times are considerably after the initial contact and exhibit the quasiperiodicity of the system. 

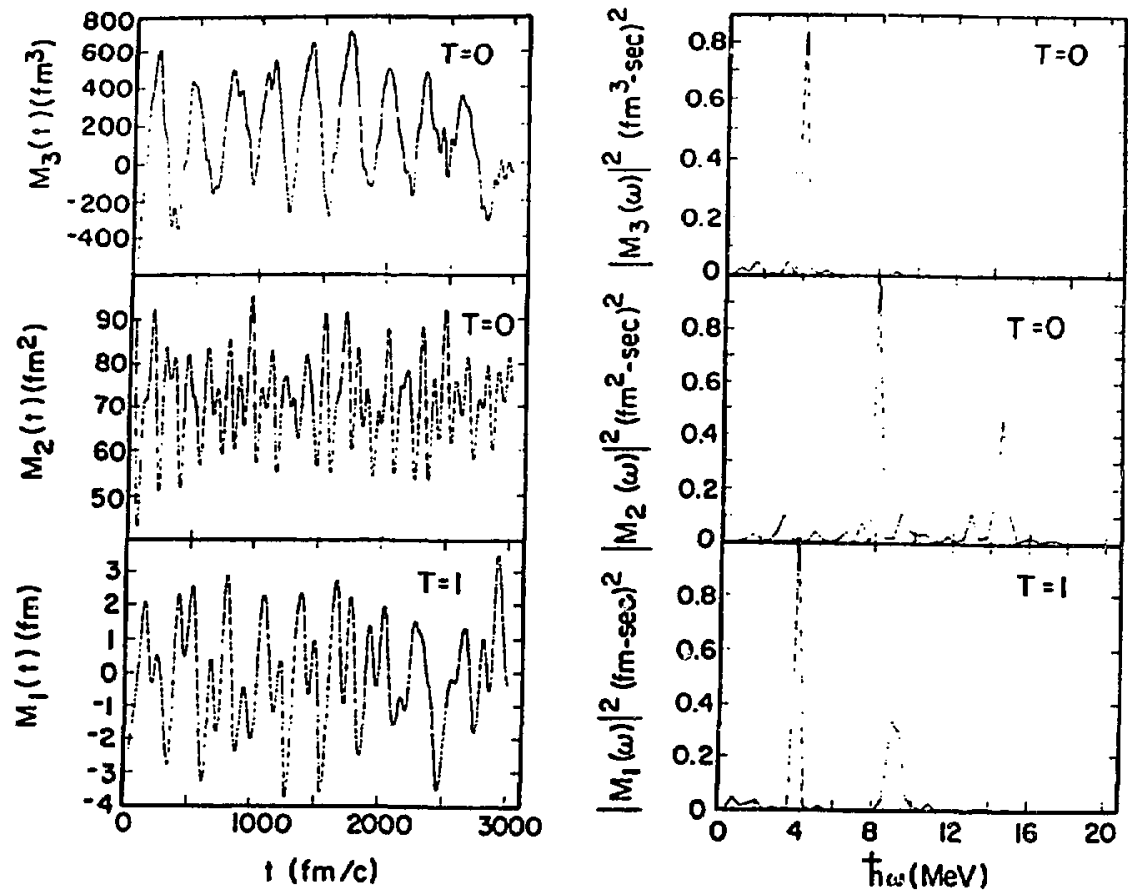

Fig. 3. The time (left column) and frequency dependency of the isovector dipole $(\ell=1)$ and the isoscalar quadrupole $(\ell=2)$ and isoscalar octupole $(\ell=3)$ moments computed from Eq. (19). The yiuantity in the figures $T=0,1$ labels the isospin which in the text is denoted by $I=0,1$.

CALCULATIONS ON CARBON CONSTRAINED HARTREE FOCK

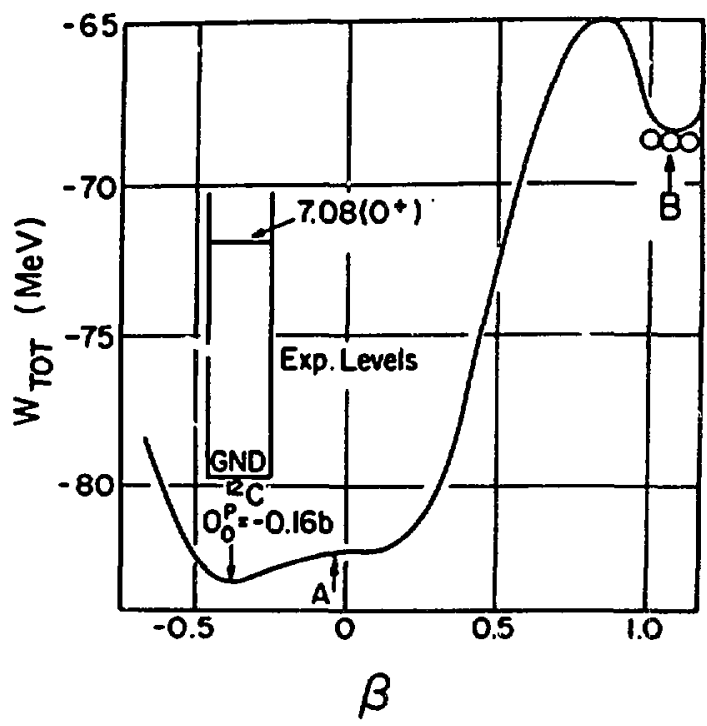

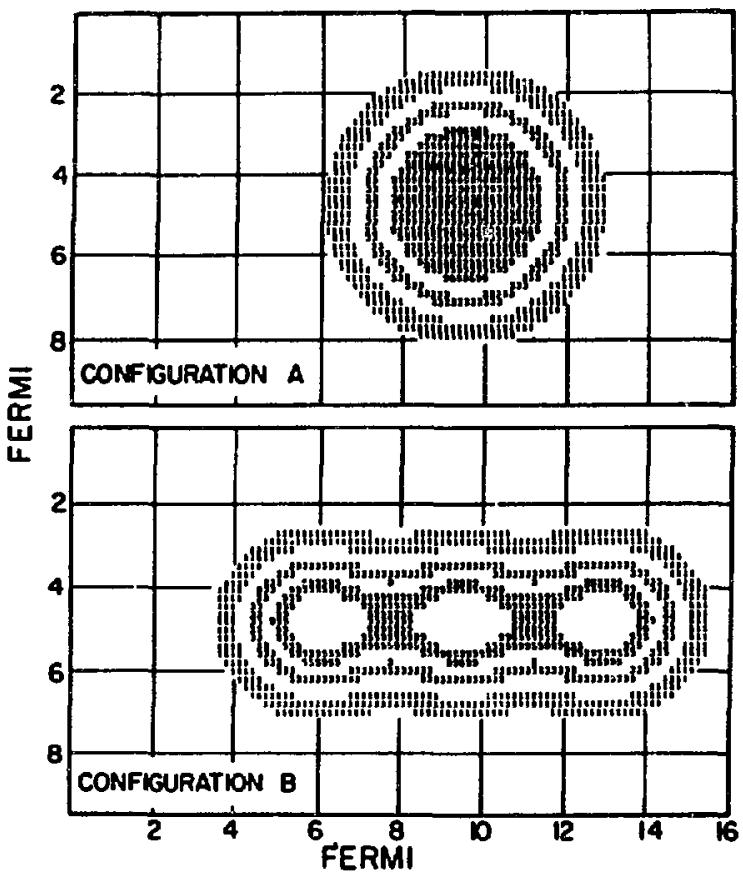

Fig. 4. Energy as a function of quadrupole deformation $\beta$ and the corresponding density configurations for ${ }^{12} \mathrm{C}$. 


$$
{ }^{12} \mathrm{C}\left(\mathrm{O}^{+}\right)+{ }^{12} \mathrm{C} \quad I=0
$$

:
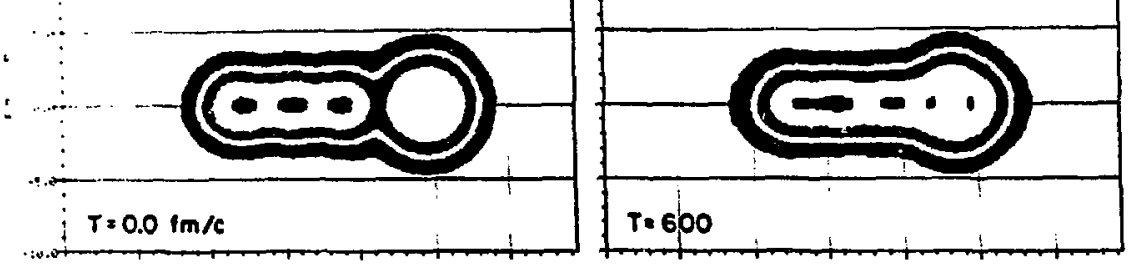

16. Then

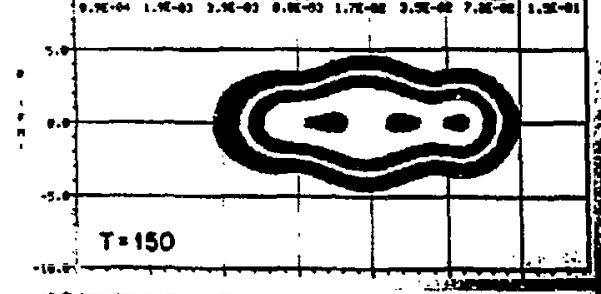

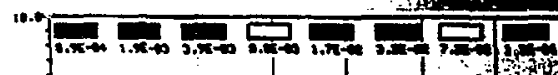

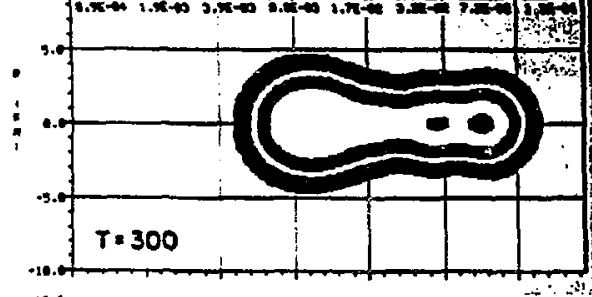

10.0 , ind

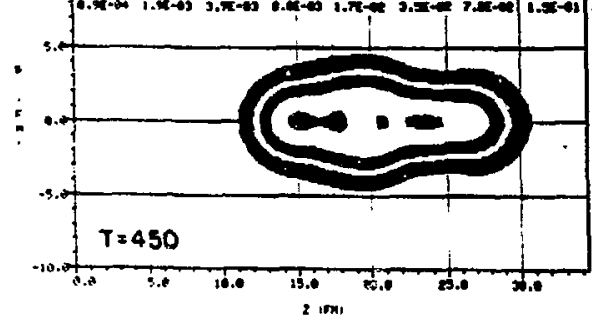

Fig. 5. The density contours of $24 \mathrm{Mg}$ evolving from a ${ }^{12} \mathrm{C}\left(0^{+}\right)+{ }^{12} \mathrm{C}$ initial configuration for the isoscalar ( $\left.\mathrm{I}=0\right)$ and iso-

vector $(I=1)$ densities.

\section{${ }^{12} \mathrm{C}\left(0+1+{ }^{12} \mathrm{C} \quad I=1\right.$}
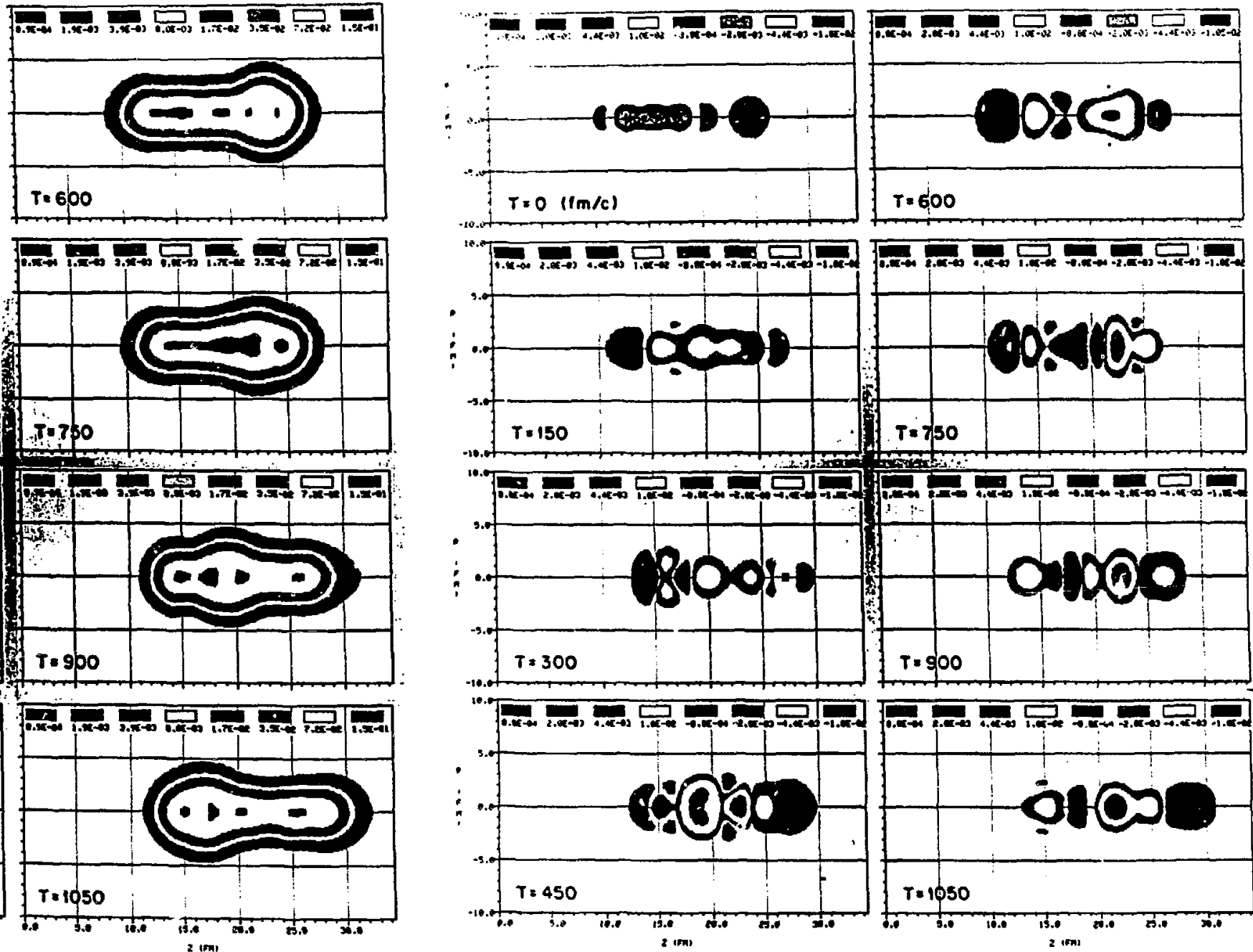
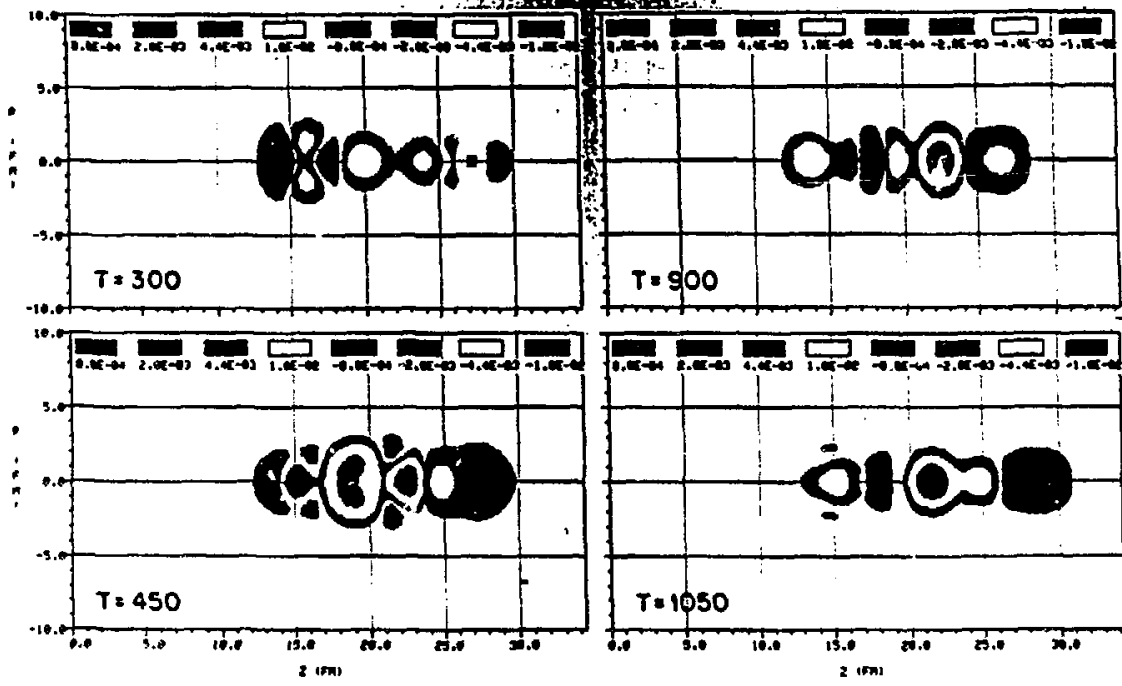
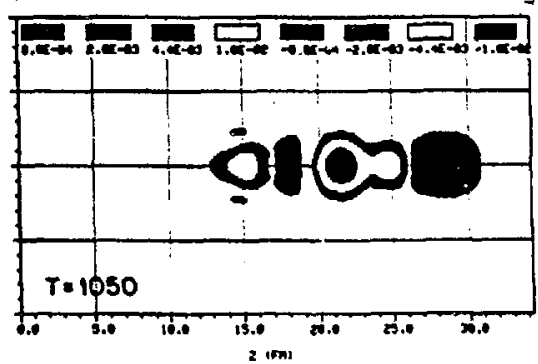


\section{${ }^{12} \mathrm{c}\left(0^{+}\right)+{ }^{12} \mathrm{c}$}
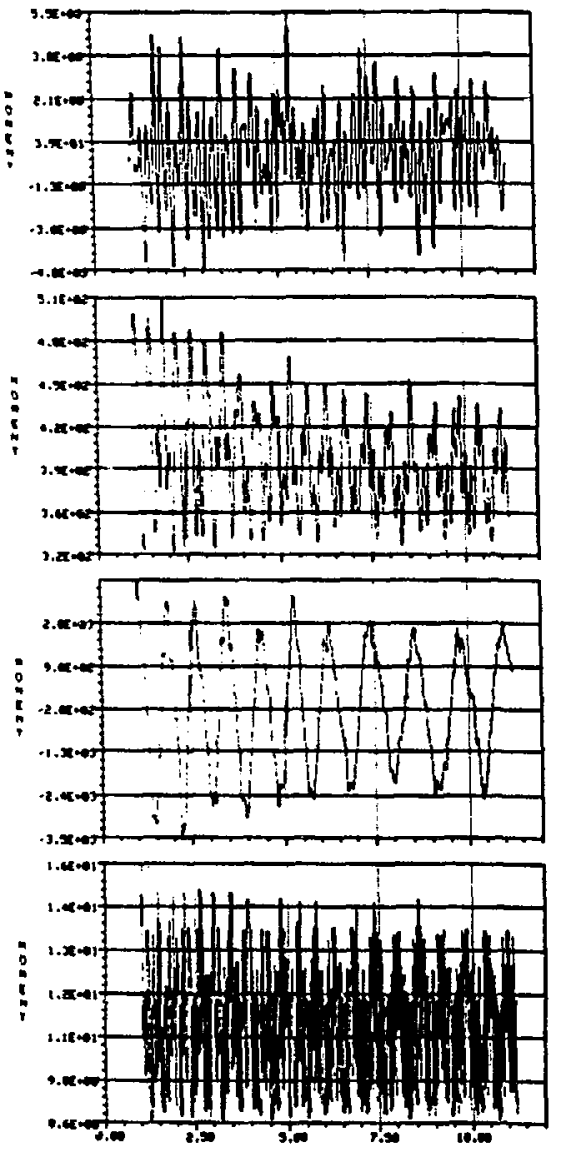

ne ituro nasi
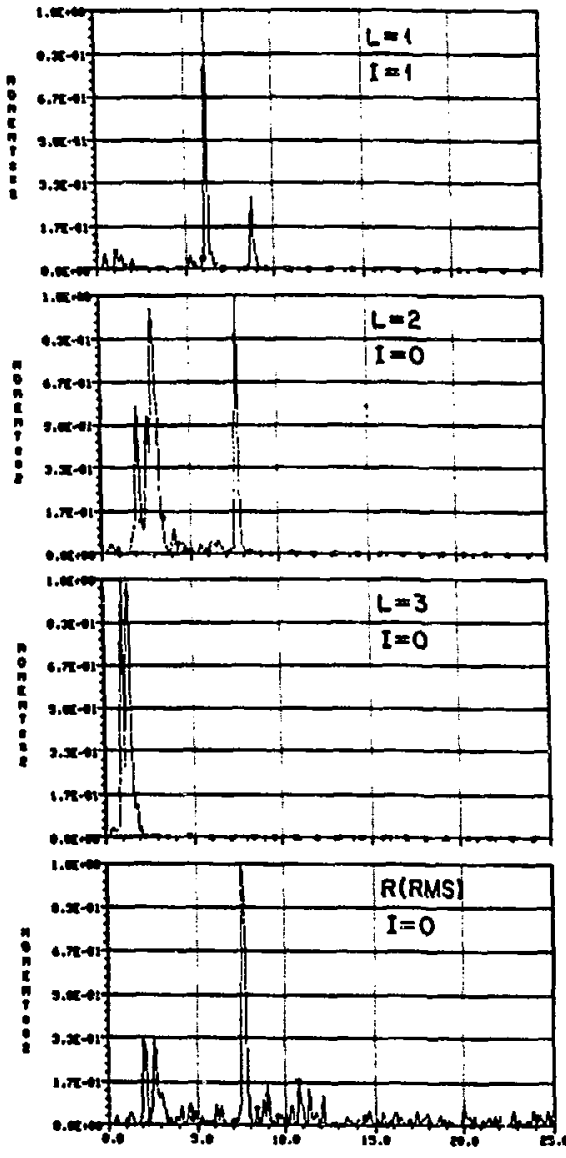

nero unour

Fig. 6. The time and frequency dependence of the isoscalar quadrupole moment, octupole moment and the RMS radius, and the isovector dipole moment for $24 \mathrm{Mg}$.

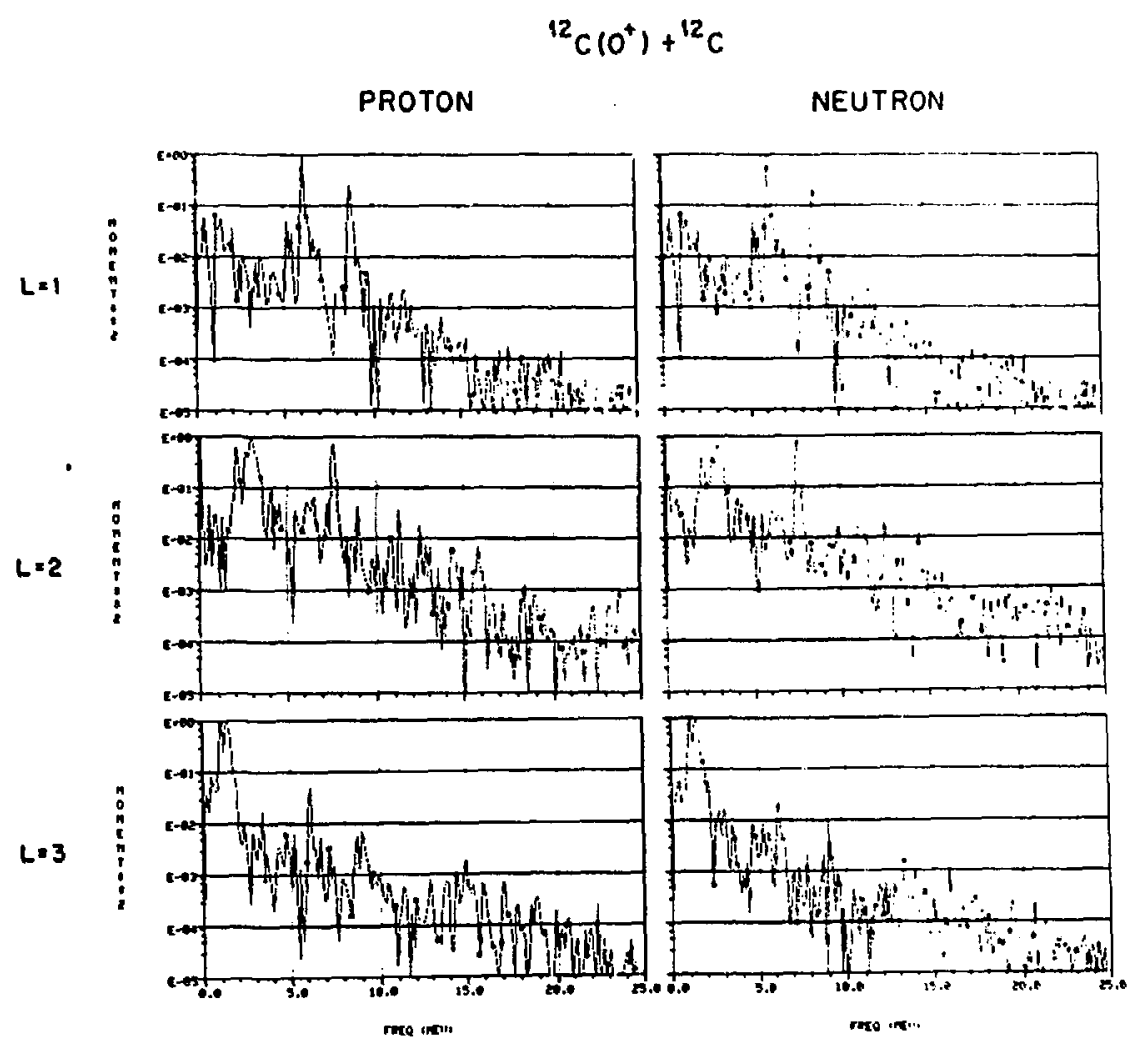

Fig. 7. The frequency dependence of the neutron and proton densities for the $24 \mathrm{Mg}$ system. 


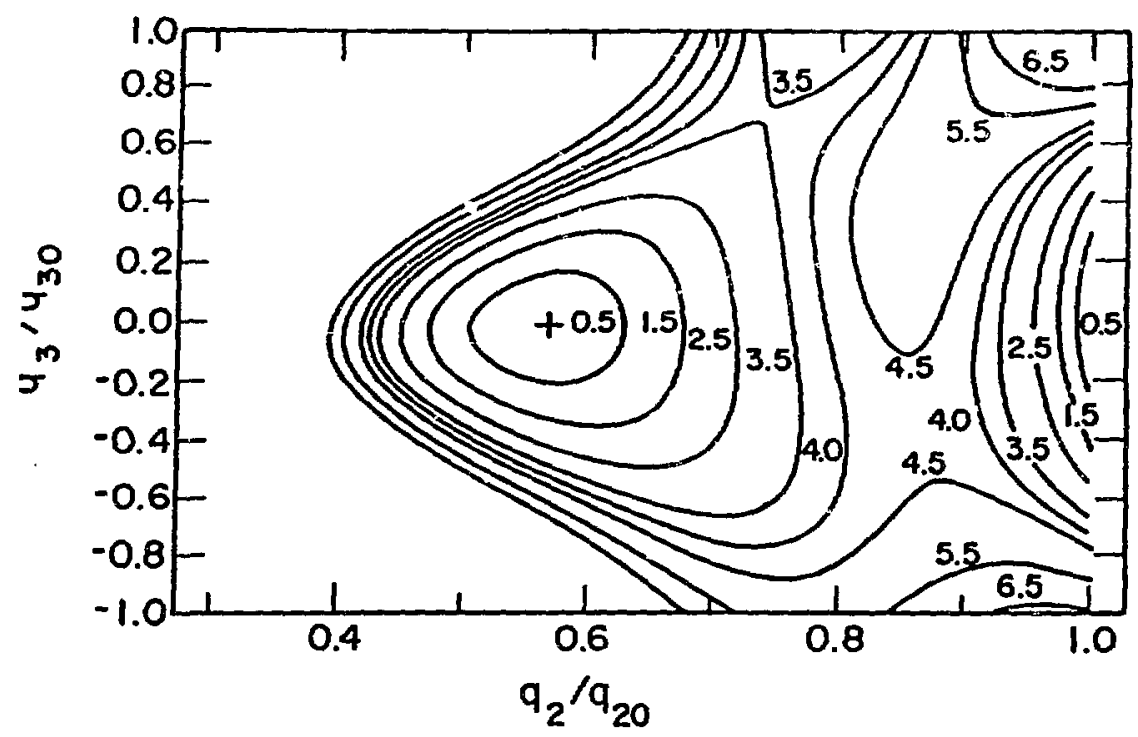

Fig. 8. Density-constrained static HF energies, $\Delta E=E$ (constrained) $\left.-E_{0}\right), E_{0}=$ $-122.3 \mathrm{MeV}$, as a function of the isoscalar quadrupole and octupole degrees of freedom. Contours are rabeled in MeV. The constants $q_{20}, q_{30}$ are $q_{20}=89.8 \mathrm{fm}^{2}$, and $q_{30}=-654.1 \mathrm{fm}^{2}$. The point having an energy $E_{0}$ is a shape isomer of the ${ }^{180}$ system.

\section{ENERGY SIIRFACE IN Q3-Q2 PLANE}

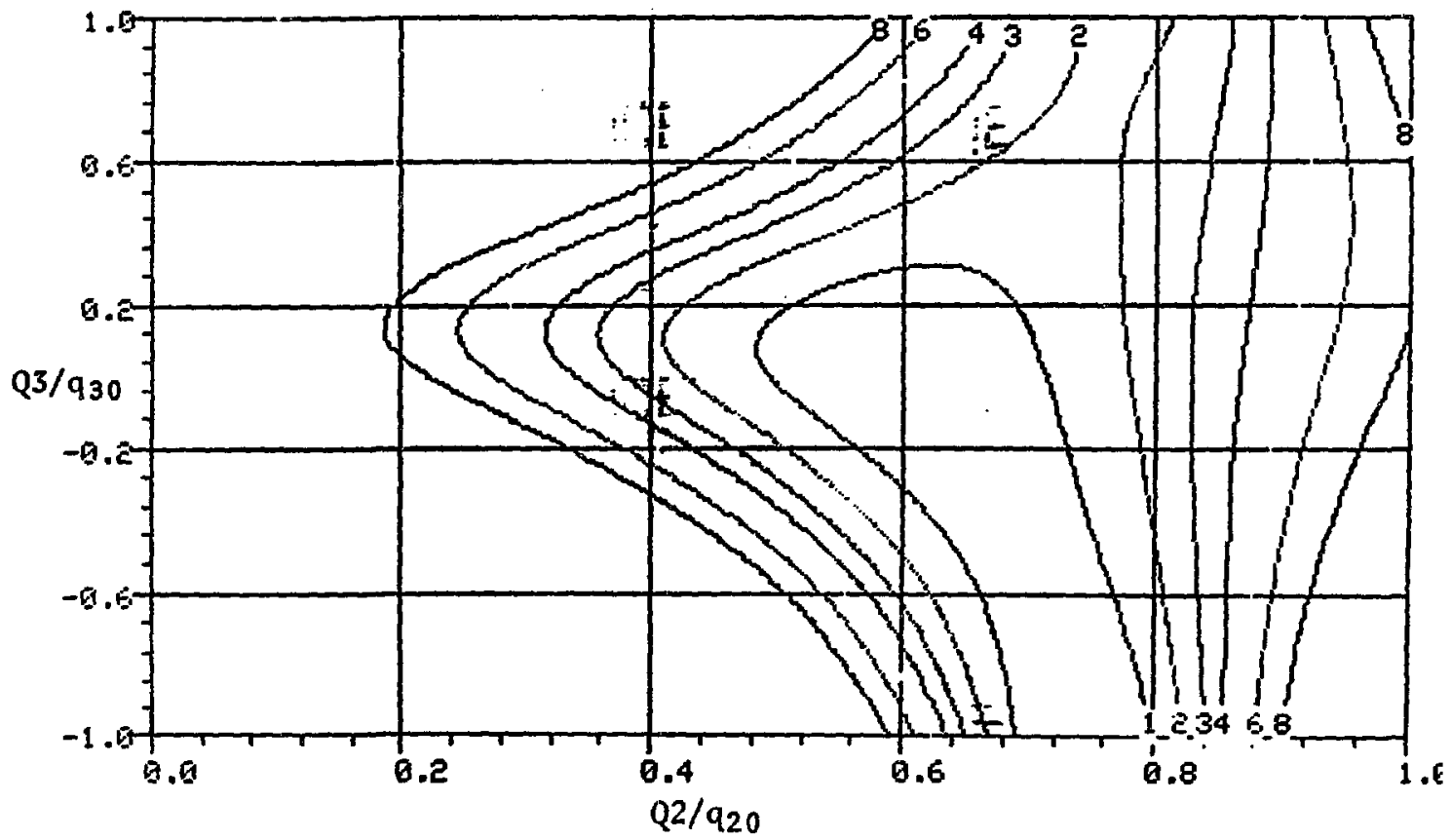

Fig. 9. Density-constrained static $H F$ energies, $\Delta E=2$ ( $E$ (constrained) $\left.-E_{0}\right), E_{0}=$ $-143.8 \mathrm{MeV}$ as a function of the isoscalar quadrupole and octupole degrees of freedom. Contours are labeled in MeV. The constants $q_{20}$ and $q_{30}$ are $q_{20}=582.7 \mathrm{fm}^{2}$ and $q_{30}$ $=3431 \mathrm{O} \mathrm{fm}^{3}$. The point having an energy $\Delta E=0$ is a shape isomer of the $24 \mathrm{Mg}$ system. 
WILLIAMS \& KOONIN, NUCL PHYS A391 (1982) 72

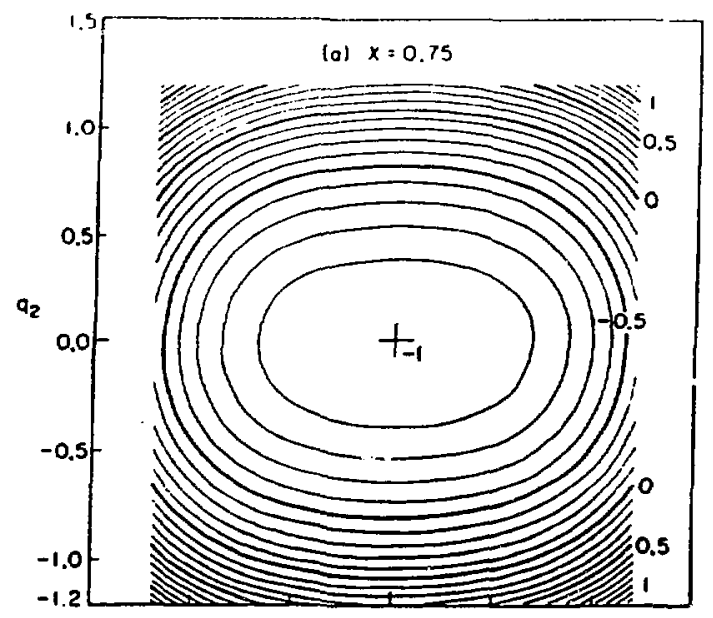

$$
\begin{aligned}
& \frac{H\left(q_{,} \rho\right)}{N \epsilon}= \\
& -1+1 / 2 q_{1}^{2}(1-x)+1 / 2 q_{2}^{2}(2-x)+1 / 2 p_{1}^{2}(1+x)+1 / 2 p_{2}^{2}(2+x) \\
& +\frac{1}{4} x\left(\left(q_{1}^{2}+q_{2}^{2}\right)^{2}-\left(p_{1}^{2}+p_{2}^{2}\right)-\left(q_{1}^{2}-p_{1}^{2}\right)\left(q_{2}^{2}-p_{2}^{2}\right)\right. \\
& \left.-4 q_{1} q_{2} p_{1} p_{2}\right)
\end{aligned}
$$
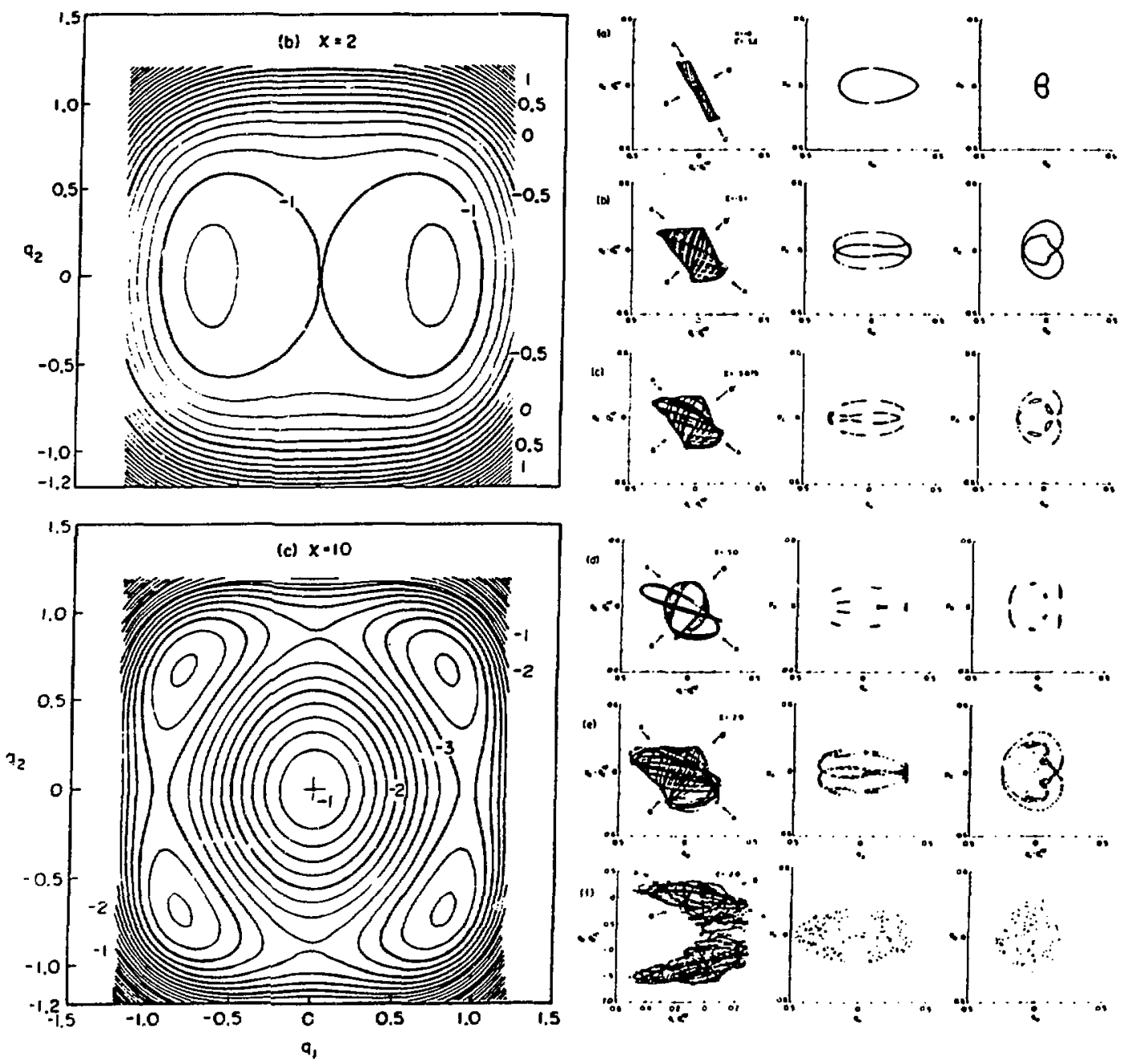

Fig. 10. The classical limit of the SU(3) Hamiltonian, the static energy surfaces for three values of the coupling constant $x$, and six trajectories and Poincaré sections, a) harmonic, b), c), d) quasiperiodic, and e), f) chaotic. 


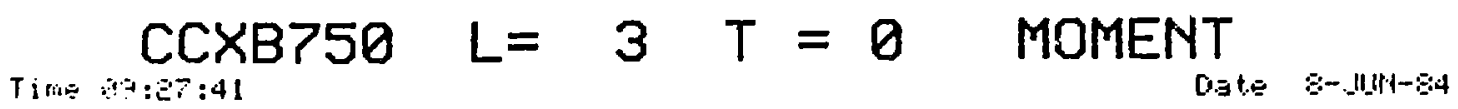

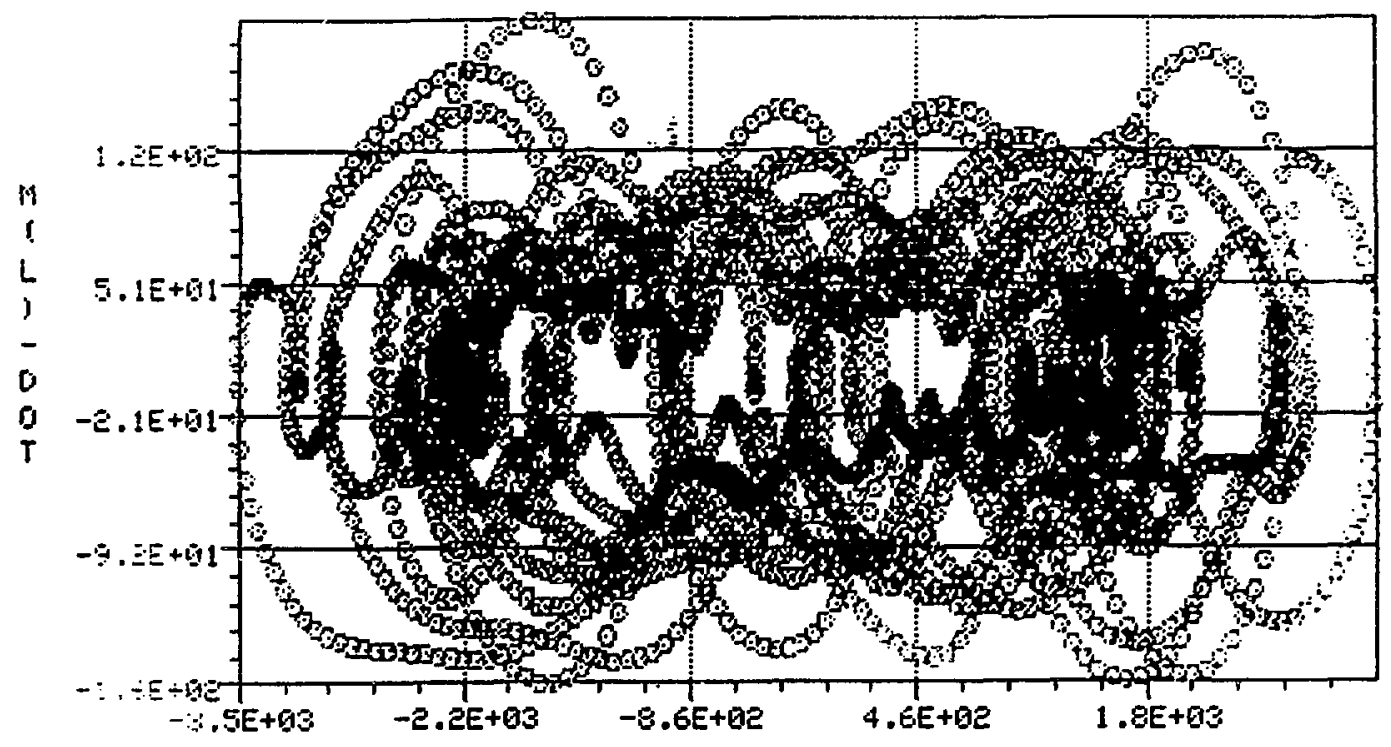

$M(L)$

Tim:

CCXB750 $L=2 T=0$ MOMENT

Date B-JIIN-84

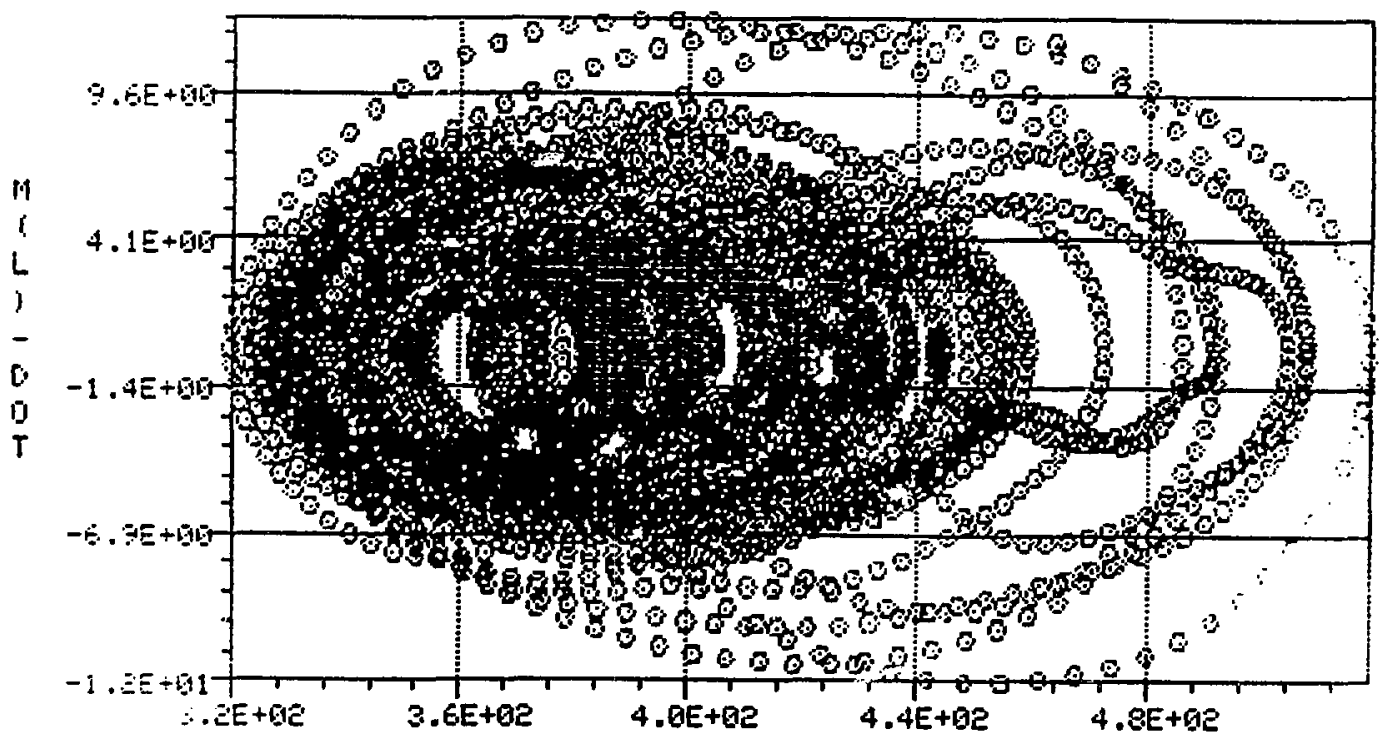

M(L)

Fig. 11. Poincaré phase space plots $M_{\ell I}(t)$ vs. $M_{\ell I}(t)$ for $I=0, \ell=2,3$. 


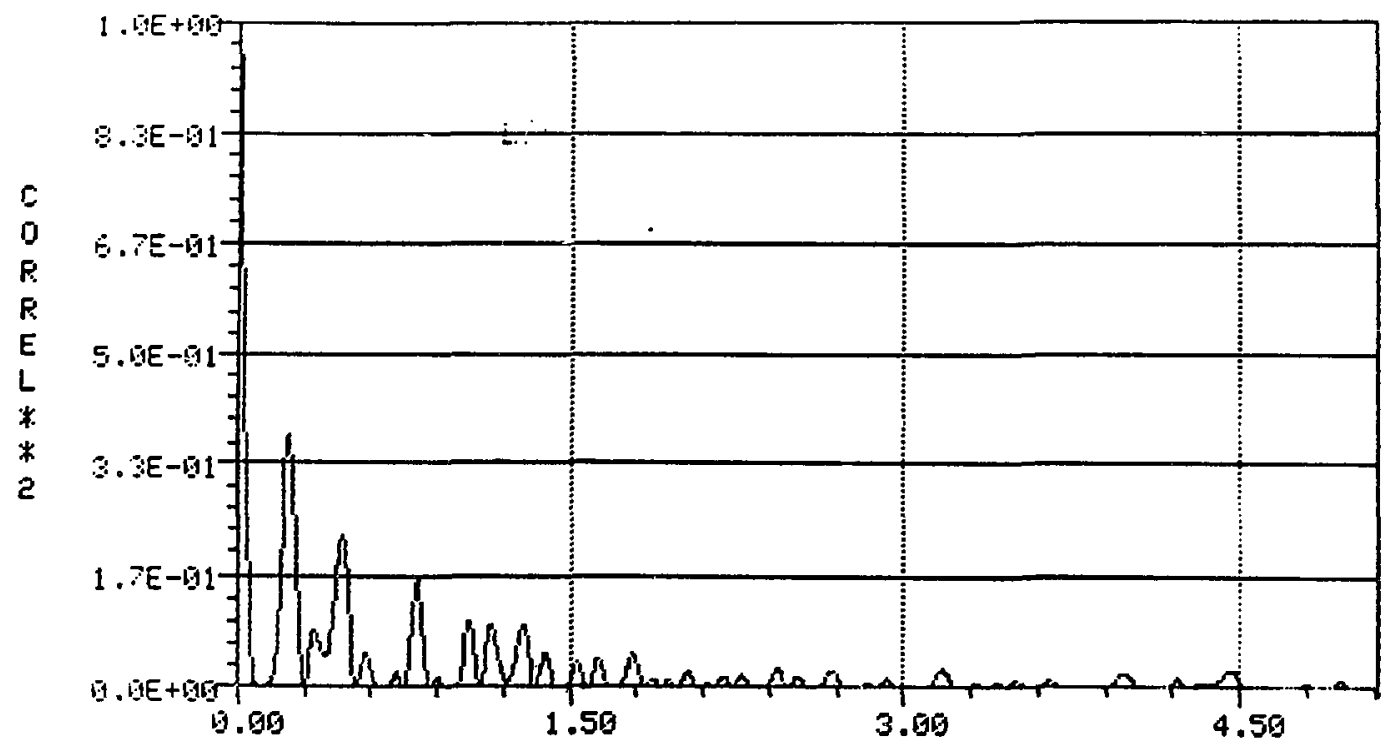

TIME (10**3 FM/C)

CCXB750 $L=3 \quad T=0$ MOMENT Time 09:48:18

Date 8-JuN-84

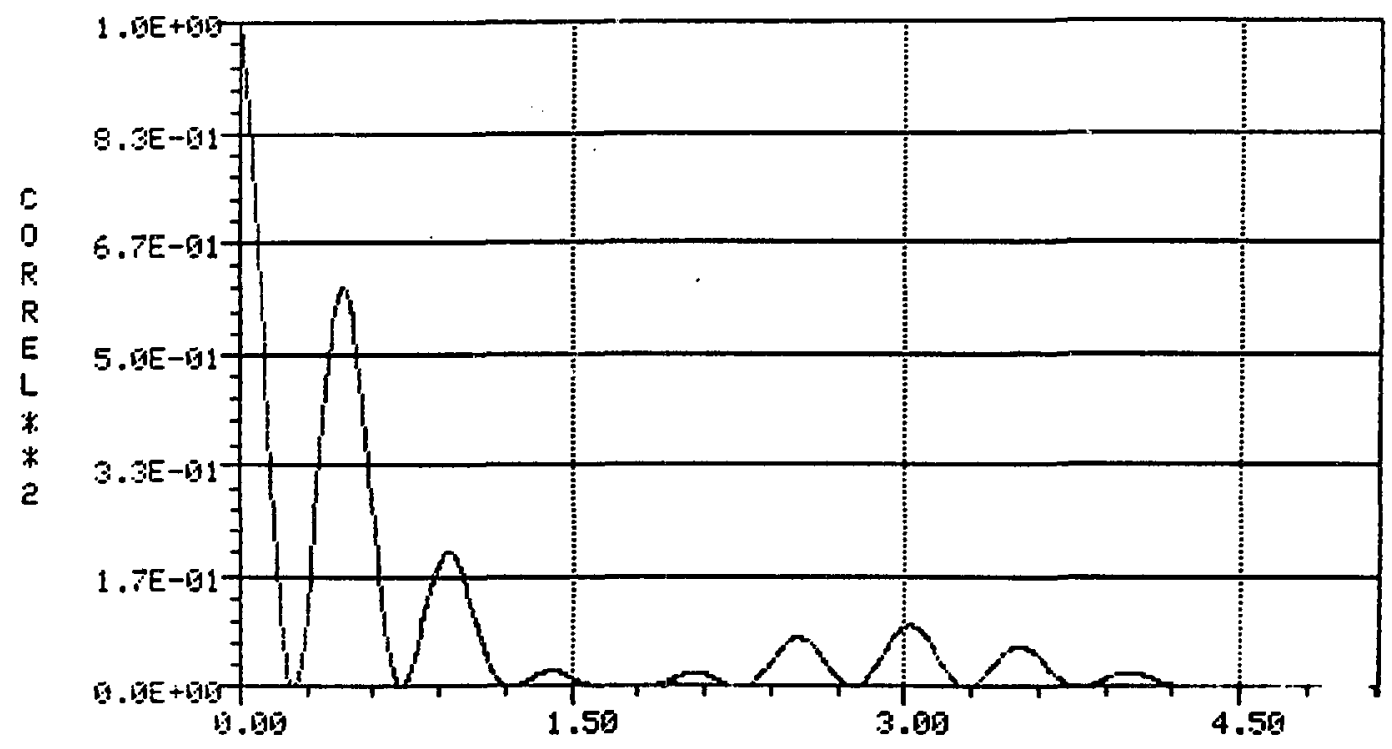

TIME (10**3 FM,C)

Fig. 12. The autocorrelation function $C_{I \ell}(t)$ as a function of time for the isoscalar quadrupole octupole modes in ${ }^{24} \mathrm{Mg}$. 
${ }^{12} \mathrm{C}\left(\mathrm{O}^{+}\right)+{ }^{12} \mathrm{C} T=0$

$L=2$
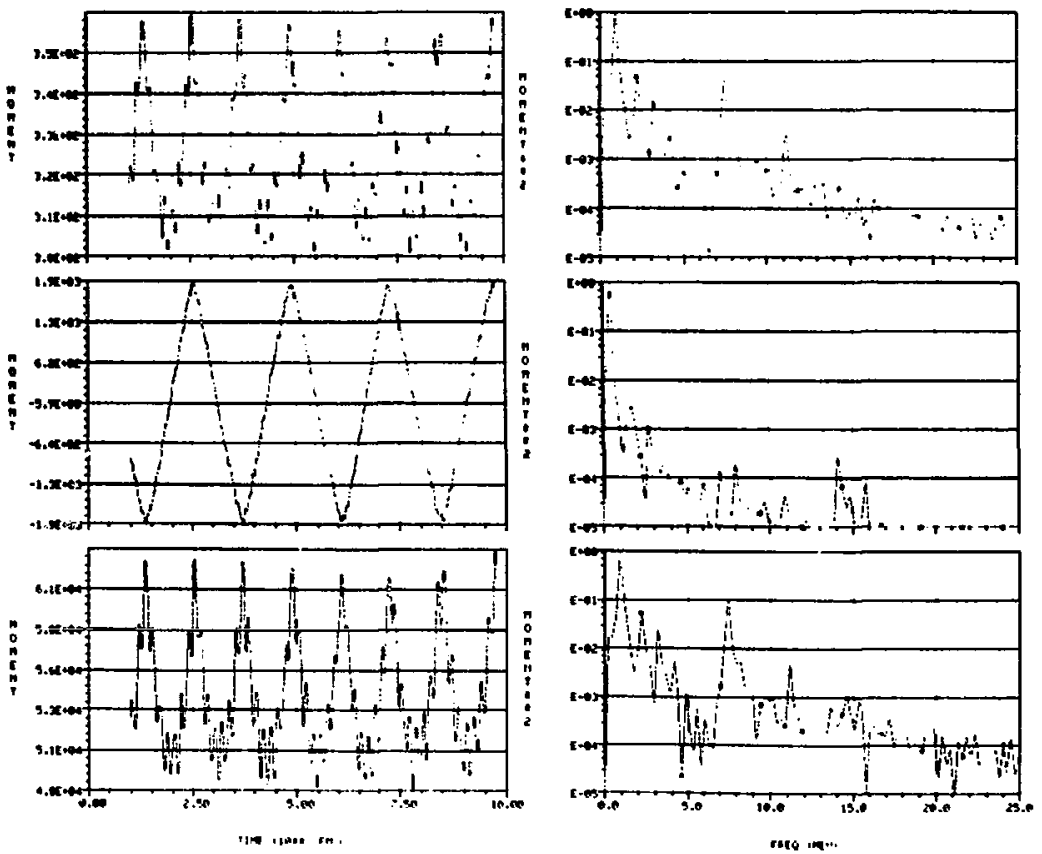

Fig. 13. Isoscalar frequency and time functions for the "cooled" modes of $24 \mathrm{Mg}$. Note the low-frequency motion is nearly harmonic.
${ }^{12} \mathrm{C}\left(0^{+}\right)+{ }^{12} \mathrm{C} T=1$

Lat
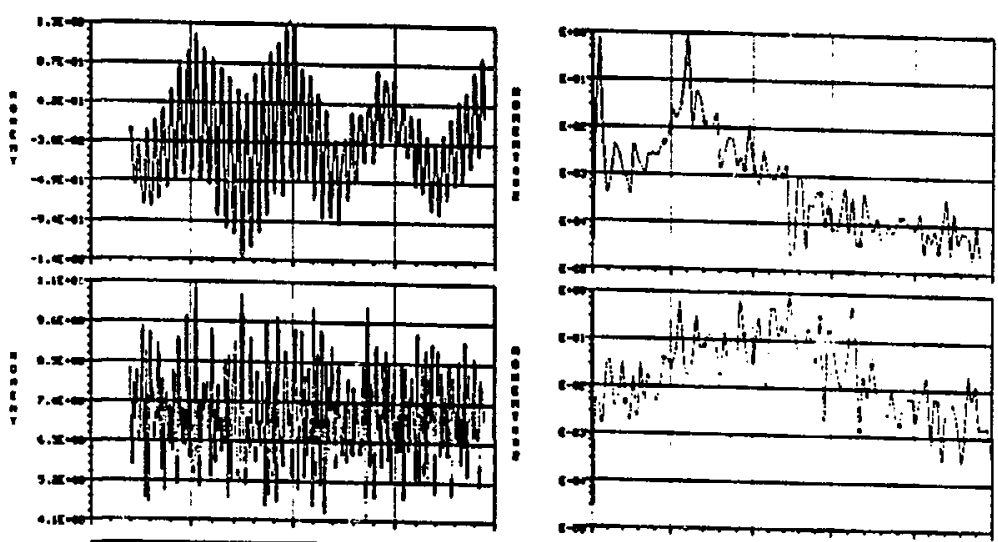

Le3
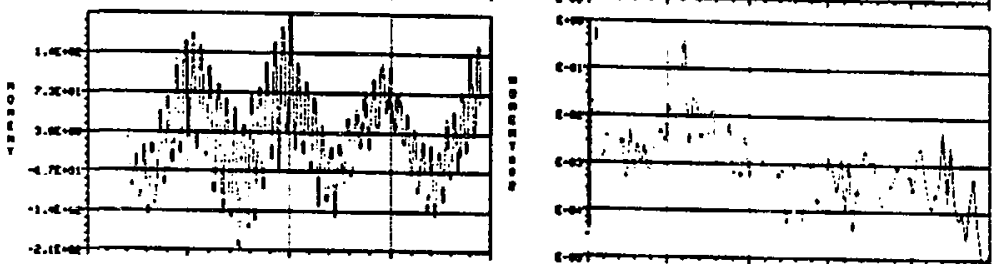

$L=4$
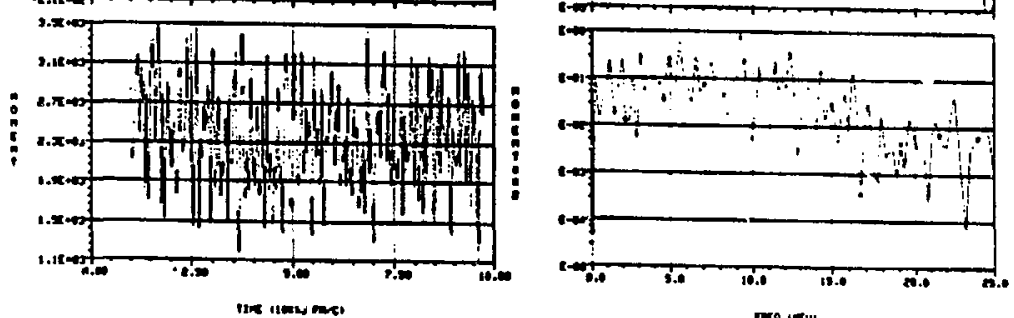

Fig. 14. Same as in Fig. 13 for the isovector modes of $24 \mathrm{Mg}$. 


$$
{ }^{12} c\left(0^{+}\right)+{ }^{12} c
$$

$L=1$
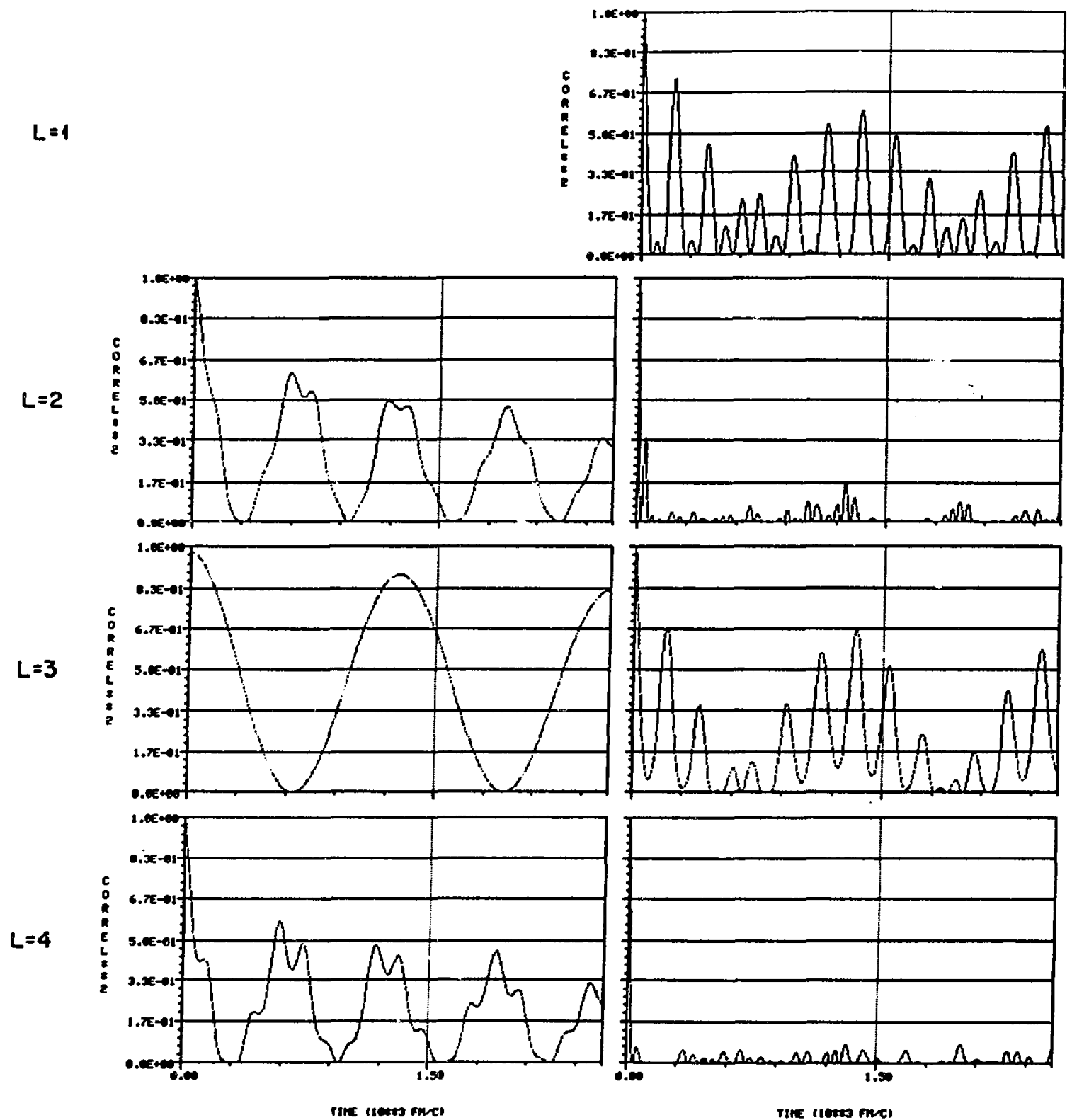

The clows fmal

Fig. 15. Autocorrelation function for the isoscalar and isovector "cooled" modes of $24 \mathrm{Mg}$. 